\title{
A Simplified Proof of Uncertainty Principle for Quaternion Linear Canonical Transform
}

\author{
Mawardi Bahri ${ }^{1}$ and Ryuichi Ashino \\ ${ }^{1}$ Department of Mathematics, Hasanuddin University, Makassar 90245, Indonesia \\ ${ }^{2}$ Division of Mathematical Sciences, Osaka Kyoiku University, Osaka 582-8582, Japan \\ Correspondence should be addressed to Ryuichi Ashino; ashino@cc.osaka-kyoiku.ac.jp
}

Received 20 August 2015; Accepted 9 December 2015

Academic Editor: Jozef Banas

Copyright (C) 2016 M. Bahri and R. Ashino. This is an open access article distributed under the Creative Commons Attribution License, which permits unrestricted use, distribution, and reproduction in any medium, provided the original work is properly cited.

We provide a short and simple proof of an uncertainty principle associated with the quaternion linear canonical transform (QLCT) by considering the fundamental relationship between the QLCT and the quaternion Fourier transform (QFT). We show how this relation allows us to derive the inverse transform and Parseval and Plancherel formulas associated with the QLCT. Some other properties of the QLCT are also studied.

\section{Introduction}

It is well-known that the traditional linear canonical transform (LCT) plays an important role in many fields of optics and signal processing. It can be regarded as a generalization of many mathematical transforms such as the Fourier transform, Laplace transform, the fractional Fourier transform, and the Fresnel transform. Many fundamental properties of this extended transform are already known, including shift, modulation, convolution, and correlation and uncertainty principle, for example, in [1-6].

Recently, there are so many studies in the literature that are concerned with the generalization of the LCT within the context of quaternion algebra, which is the so-called quaternion linear canonical transform (QLCT) (see, e.g., [710]). They also established some important properties of the QLCT such as inversion formula and the uncertainty principle. An application of the QLCT to study of generalized swept-frequency filters was presented in [11]. In this paper, we will focus on the two-dimensional case and provide a new proof of uncertainty principle associated with the QLCT, the ones proposed in [8], the proof of which is much simpler using the component-wise and directional uncertainty principles for the QFT $[12,13]$. Therefore, before proving this main result, we first derive the fundamental relationship between the QLCT and QFT. Using the relation, we obtain useful properties of the QLCT such as inverse transform and Parseval formula associated with the QLCT.

The quaternion algebra over $\mathbb{R}$, denoted by $\mathbb{\sharp}$, is an associative noncommutative four-dimensional algebra:

$$
\mathbb{H}=\left\{q=q_{0}+\mathbf{i} q_{1}+\mathbf{j} q_{2}+\mathbf{k} q_{3} ; q_{0}, q_{1}, q_{2}, q_{3} \in \mathbb{R}\right\},
$$

which obeys the following multiplication rules:

$$
\begin{aligned}
\mathbf{i j} & =-\mathbf{j i}=\mathbf{k}, \\
\mathbf{j k} & =-\mathbf{k} \mathbf{j}=\mathbf{i}, \\
\mathbf{k} \mathbf{i} & =-\mathbf{i k}=\mathbf{j}, \\
\mathbf{i}^{2} & =\mathbf{j}^{2}=\mathbf{k}^{2}=\mathbf{i j k}=-1 .
\end{aligned}
$$

For a quaternion $q=q_{0}+\mathbf{i} q_{1}+\mathbf{j} q_{2}+\mathbf{k} q_{3} \in \mathbb{U}, q_{0}$ is called the scalar part of $q$ denoted by $\mathrm{Sc}(q)$ and $\mathbf{i} q_{1}+\mathbf{j} q_{2}+\mathbf{k} q_{3}$ is called the vector (or pure) part of $q$. The vector part of $q$ is conventionally denoted by $\mathbf{q}$. Let $p, q \in \mathbb{U}$ and $\mathbf{p}, \mathbf{q}$ be their vector parts, respectively. Equation (2) yields the quaternionic multiplication $q p$ as

$$
q p=q_{0} p_{0}-\mathbf{q} \cdot \mathbf{p}+q_{0} \mathbf{p}+p_{0} \mathbf{q}+\mathbf{q} \times \mathbf{p},
$$

where $\mathbf{q} \cdot \mathbf{p}=\left(q_{1} p_{1}+q_{2} p_{2}+q_{3} p_{3}\right)$ and $\mathbf{q} \times \mathbf{p}=\mathbf{i}\left(q_{2} p_{3}-q_{3} p_{2}\right)+$ $\mathbf{j}\left(q_{3} p_{1}-q_{1} p_{3}\right)+\mathbf{k}\left(q_{1} p_{2}-q_{2} p_{1}\right)$. 
The quaternion conjugate of $q$, given by

$$
\bar{q}=q_{0}-\mathbf{i} q_{1}-\mathbf{j} q_{2}-\mathbf{k} q_{3}, \quad q_{0}, q_{1}, q_{2}, q_{3} \in \mathbb{R},
$$

is an anti-involution; that is,

$$
\bar{q} \bar{p}=\bar{p} \bar{q}
$$

From (4) we obtain the norm or modulus of $q \in \mathbb{W}$ defined as

$$
|q|=\sqrt{q \bar{q}}=\sqrt{q_{0}^{2}+q_{1}^{2}+q_{2}^{2}+q_{3}^{2}} .
$$

It is not difficult to see that

$$
|q p|=|q||p|, \quad \forall p, q \in \mathbb{H} .
$$

Furthermore, it is easily seen that

$$
|p q r|=|r q p|, \quad \forall p, q, r \in \mathbb{H} .
$$

Using conjugate (4) and the modulus of $q$, we can define the inverse of $q \in \mathbb{H} \backslash\{0\}$ as

$$
q^{-1}=\frac{\bar{q}}{|q|^{2}}
$$

which shows that $\mathbb{W}$ is a normed division algebra.

It is convenient to introduce an inner product for quaternion-valued (in the rest of the paper, we will always consider quaternion function) functions $f, g: \mathbb{R}^{2} \rightarrow \mathbb{H}$ as

$$
(f, g)=\int_{\mathbb{R}^{2}} f(\mathbf{x}) \overline{g(\mathbf{x})} d \mathbf{x}, \quad d \mathbf{x}=d x_{1} d x_{2},
$$

with symmetric real scalar part

$$
\langle f, g\rangle=\frac{1}{2}[(f, g)+(g, f)]=\mathrm{Sc} \int_{\mathbb{R}^{2}} f(\mathbf{x}) \bar{g}(\mathbf{x}) d \mathbf{x} .
$$

In particular, for $f=g$, we obtain the $L^{2}\left(\mathbb{R}^{2} ; \mathbb{H}\right)$-norm:

$$
\|f\|=\sqrt{\langle f, f\rangle}=\left(\int_{\mathbb{R}^{2}}|f(\mathbf{x})|^{2} d^{2} \mathbf{x}\right)^{1 / 2} .
$$

\section{Quaternion Linear Canonical Transform}

In this section we begin by defining the two-sided QFT (for simplicity of notation we write the QFT instead of the twosided QFT in the next section). We discus some properties, which will be used to prove the uncertainty principle.

Definition 1. The QFT of $f \in L^{1}\left(\mathbb{R}^{2} ; \mathbb{U}\right)$ is the transform $\mathscr{F}_{q}\{f\}: \mathbb{R}^{2} \rightarrow \mathbb{M}$ given by the integral

$$
\mathscr{F}_{q}\{f\}(\boldsymbol{\omega})=\frac{1}{\sqrt{(2 \pi)^{2}}} \int_{\mathbb{R}^{2}} e^{-\mathbf{i} \omega_{1} x_{1}} f(\mathbf{x}) e^{-\mathbf{j} \omega_{2} x_{2}} d \mathbf{x},
$$

where $\mathbf{x}=x_{1} \mathbf{e}_{1}+x_{2} \mathbf{e}_{2}, \boldsymbol{\omega}=\omega_{1} \mathbf{e}_{1}+\omega_{2} \mathbf{e}_{2}$, and the quaternion exponential product $e^{-\mathbf{i} \omega_{1} x_{1}} e^{-\mathbf{j} \omega_{2} x_{2}}$ is the quaternion Fourier kernel. Here $\mathscr{F}_{q}$ is called the quaternion Fourier transform operator.
Definition 2. If $f \in L^{1}\left(\mathbb{R}^{2} ; \mathbb{M}\right)$ and $\mathscr{F}_{q}\{f\} \in L^{1}\left(\mathbb{R}^{2} ; \mathbb{M}\right)$, then the inverse transform of the QFT is given by

$$
\begin{aligned}
f(\mathbf{x}) & =\mathscr{F}_{q}^{-1}\left[\mathscr{F}_{q}\{f\}\right](\mathbf{x}) \\
& =\frac{1}{\sqrt{(2 \pi)^{2}}} \int_{\mathbb{R}^{2}} e^{\mathbf{i} \omega_{1} x_{1}} \mathscr{F}_{q}\{f\}(\boldsymbol{\omega}) e^{\mathbf{j} \omega_{2} x_{2}} d \boldsymbol{\omega},
\end{aligned}
$$

where $\mathscr{F}_{q}^{-1}$ is called the inverse QFT operator.

An important property of the QFT is stated in the following lemma, which is needed to prove Parseval formula of the QLCT. For more details of the QFT, see [12-16].

Lemma 3 (QFT Parseval). The quaternion product of $f, g \in$ $L^{1}\left(\mathbb{R}^{2} ; \mathbb{H}\right) \cap L^{2}\left(\mathbb{R}^{2} ; \mathbb{H}\right)$ and its QFT are related by

$$
\langle f, g\rangle_{L^{2}\left(\mathbb{R}^{2} ; \mathbb{H}\right)}=\left\langle\mathscr{F}_{q}\{f\}, \mathscr{F}_{q}\{g\}\right\rangle_{L^{2}\left(\mathbb{R}^{2} ; \mathbb{H}\right)} .
$$

In particular, with $f=g$, we get the quaternion version of the Plancherel formula; that is,

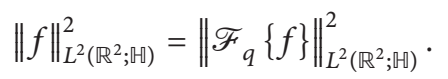

Based on the definition of the QFT mentioned above, we consider the two-sided QLCT which is defined as follows.

Definition 4 (QLCT). Let $A_{1}=\left(a_{1}, b_{1}, c_{1}, d_{1}\right)$ and $A_{2}=$ $\left(a_{2}, b_{2}, c_{2}, d_{2}\right)$ be two matrix parameters satisfying $\operatorname{det}\left(A_{s}\right)=$ $a_{s} d_{s}-b_{s} c_{s}=1, s=1,2$. The QLCT of a quaternion signal $f \in L^{1}\left(\mathbb{R}^{2} ; \mathbb{H}\right)$ is defined by

$$
\begin{aligned}
& L_{A_{1}, A_{2}}^{\mathbb{\sharp}}\{f\}(\boldsymbol{\omega}) \\
& = \begin{cases}\int_{\mathbb{R}^{2}} K_{A_{1}}\left(x_{1}, \omega_{1}\right) f(\mathbf{x}) K_{A_{2}}\left(x_{2}, \omega_{2}\right) d \mathbf{x}, & b_{1} b_{2} \neq 0 \\
\sqrt{d_{1}} e^{\mathrm{i}\left(c_{1} d_{1} / 2\right) \omega_{1}^{2}} f\left(d_{1} \omega_{1}, x_{2}\right) K_{A_{2}}\left(x_{2}, \omega_{2}\right), & b_{1}=0, \quad b_{2} \neq 0 \\
\sqrt{d_{2}} e^{\mathrm{j}\left(c_{2} d_{2} / 2\right) \omega_{2}^{2}} f\left(x_{1}, d_{2} \omega_{2}\right) K_{A_{1}}\left(x_{1}, \omega_{1}\right), & b_{1} \neq 0, \quad b_{2}=0 \\
\sqrt{d_{1} d_{2}} e^{\mathrm{i}\left(c_{1} d_{1} / 2\right) \omega_{1}^{2}} f\left(d_{1} \omega_{1}, d_{2} \omega_{2}\right) e^{\mathrm{j}\left(c_{2} d_{2} / 2\right) \omega_{2}^{2}}, & b_{1}=b_{2}=0,\end{cases}
\end{aligned}
$$

where the kernel functions of the QLCT are given by, respectively,

$$
\begin{aligned}
& K_{A_{1}}\left(x_{1}, \omega_{1}\right) \\
& \quad=\frac{1}{\sqrt{2 \pi b_{1}}} e^{\mathbf{i}(1 / 2)\left(\left(a_{1} / b_{1}\right) x_{1}^{2}-\left(2 / b_{1}\right) x_{1} \omega_{1}+\left(d_{1} / b_{1}\right) \omega_{1}^{2}-(\pi / 2)\right)},
\end{aligned}
$$

$$
b_{1} \neq 0
$$

$$
\begin{aligned}
& K_{A_{2}}\left(x_{2}, \omega_{2}\right) \\
& =\frac{1}{\sqrt{2 \pi b_{2}}} e^{\mathbf{j}(1 / 2)\left(\left(a_{2} / b_{2}\right) x_{2}^{2}-\left(2 / b_{2}\right) x_{2} \omega_{2}+\left(d_{2} / b_{2}\right) \omega_{2}^{2}-(\pi / 2)\right)},
\end{aligned}
$$

$$
b_{2} \neq 0 \text {. }
$$

From the definition of the QLCT, we can see easily that when $b_{1} b_{2}=0$ and $b_{1}=b_{2}=0$, the QLCT of a signal 
is essentially a quaternion chirp multiplication. Therefore, in this work we always assume that $b_{1} b_{2} \neq 0$. As a special case, when $A_{1}=A_{2}=\left(a_{i}, b_{i}, c_{i}, d_{i}\right)=(0,1,-1,0)$ for $i=1,2$, LCT definition (17) reduces to the QFT definition. That is,

$$
\begin{aligned}
& L_{A_{1}, A_{2}}^{\mathbb{\Perp}}\{f\}(\boldsymbol{\omega}) \\
& \quad=\int_{\mathbb{R}^{2}} \frac{e^{-\mathbf{i}(\pi / 4)}}{\sqrt{2 \pi}} f(\mathbf{x}) e^{-\mathbf{i} \omega_{1} x_{1}} \frac{e^{-\mathbf{j}(\pi / 4)}}{\sqrt{2 \pi}} e^{-\mathbf{j} \omega_{2} x_{2}} d \mathbf{x} \\
& \quad=e^{-\mathbf{i}(\pi / 4)} \mathscr{F}_{q}\{f\}(\boldsymbol{\omega}) e^{-\mathbf{j}(\pi / 4)},
\end{aligned}
$$

where $\mathscr{F}_{q}\{f\}$ is the QFT of $f$ given by (13).

We need the following important result (compare to [17, 18]), which will be useful in proving Theorem 15 .

Theorem 5. The QLCT of a quaternion function $f \in$ $L^{1}\left(\mathbb{R}^{2} ; \mathbb{U}\right)$ with matrix parameters $A_{1}=\left(a_{1}, b_{1}, c_{1}, d_{1}\right)$ and $A_{2}=\left(a_{2}, b_{2}, c_{2}, d_{2}\right)$ can be reduced to the $Q F T$

$$
\mathscr{F}_{q}\left\{g_{f}\right\}(\boldsymbol{\omega})=\frac{1}{\sqrt{(2 \pi)^{2}}} \int_{\mathbb{R}^{2}} e^{-\mathbf{i} x_{1} \omega_{1}} g_{f}(\mathbf{x}) e^{-\mathbf{j} x_{2} \omega_{2}} d \mathbf{x},
$$

where

$$
\begin{aligned}
\mathscr{F}_{q}\left\{g_{f}\right\}(\boldsymbol{\omega}) & =\widetilde{\mathscr{F}}(\mathbf{b} \boldsymbol{\omega}), \\
g_{f}(\mathbf{x}) & =\frac{e^{-\mathbf{i}(\pi / 4)}}{\sqrt{b_{1}}} \widetilde{f}(\mathbf{x}) \frac{e^{-\mathbf{j}(\pi / 4)}}{\sqrt{b_{2}}}, \\
\widetilde{f}(\mathbf{x}) & =e^{\mathbf{i}\left(a_{1} / 2 b_{1}\right) x_{1}^{2}} f(\mathbf{x}) e^{\mathbf{j}\left(a_{2} / 2 b_{2}\right) x_{2}^{2}},
\end{aligned}
$$

with

$$
\begin{aligned}
& \widetilde{\mathscr{F}}(\boldsymbol{\omega})=\frac{1}{\sqrt{(2 \pi)^{2}}} \int_{\mathbb{R}^{2}} e^{-\mathbf{i} x_{1}\left(\omega_{1} / b_{1}\right)} g_{f}(\mathbf{x}) e^{-\mathbf{j} x_{2}\left(\omega_{2} / b_{2}\right)} d \mathbf{x}, \\
& \widetilde{\mathscr{F}}(\boldsymbol{\omega})=e^{-\mathbf{i}\left(d_{1} / 2 b_{1}\right) \omega_{1}^{2}} L_{A_{1}, A_{2}}^{\mathbb{Q}}\{f\}(\boldsymbol{\omega}) e^{-\mathbf{j}\left(d_{2} / 2 b_{2}\right) \omega_{2}^{2}} .
\end{aligned}
$$

Proof. Simple computations using Definition 4 show that

$$
\begin{aligned}
& L_{A_{1}, A_{2}}^{\llbracket}\{f\}(\boldsymbol{\omega})=\frac{1}{\sqrt{2 \pi b_{1}}} \\
& \cdot \int_{\mathbb{R}^{2}} e^{\mathbf{i}(1 / 2)\left(\left(a_{1} / b_{1}\right) x_{1}^{2}-\left(2 / b_{1}\right) x_{1} \omega_{1}+\left(d_{1} / b_{1}\right) \omega_{1}^{2}-(\pi / 2)\right)} f(\mathbf{x}) \\
& \cdot \frac{1}{\sqrt{2 \pi b_{2}}} e^{\mathbf{j}(1 / 2)\left(\left(a_{2} / b_{2}\right) x_{2}^{2}-\left(2 / b_{2}\right) x_{2} \omega_{2}+\left(d_{2} / b_{2}\right) \omega_{2}^{2}-(\pi / 2)\right)} d \mathbf{x} \\
& =\frac{e^{-\mathbf{i}(\pi / 4)}}{\sqrt{2 \pi b_{1}}} e^{\mathbf{i}\left(d_{1} / 2 b_{1}\right) \omega_{1}^{2}} \int_{\mathbb{R}^{2}} e^{-\mathbf{i} x_{1}\left(\omega_{1} / b_{1}\right)}\left(e^{\mathbf{i}\left(a_{1} / 2 b_{1}\right) x_{1}^{2}} f(\mathbf{x})\right. \\
& \left.\cdot e^{\mathbf{j}\left(a_{2} / 2 b_{2}\right) x_{2}^{2}}\right) \frac{e^{-\mathbf{j}(\pi / 4)}}{\sqrt{2 \pi b_{2} \mathbf{j}}} e^{-\mathbf{j} x_{2}\left(\omega_{2} / b_{2}\right)} e^{\mathbf{j}\left(d_{2} / 2 b_{2}\right) \omega_{2}^{2}} d \mathbf{x} \\
& =\frac{e^{-\mathbf{i}(\pi / 4)}}{\sqrt{2 \pi b_{1}}} e^{\mathbf{i}\left(d_{1} / 2 b_{1}\right) \omega_{1}^{2}} \int_{\mathbb{R}^{2}} e^{-\mathbf{i} x_{1}\left(\omega_{1} / b_{1}\right)} \tilde{f}(\mathbf{x}) \\
& \cdot e^{-\mathbf{j} x_{2}\left(\omega_{2} / b_{2}\right)} d \mathbf{x} \frac{e^{-\mathbf{i}(\pi / 4)}}{\sqrt{2 \pi b_{2}}} e^{\mathbf{e}^{-\mathbf{j}(\pi / 4)}\left(d_{2} / 2 b_{2}\right) \omega_{2}^{2}} \cdot
\end{aligned}
$$

Then, multiplying both sides of (24) by $e^{-\mathbf{i}\left(d_{1} / 2 b_{1}\right) \omega_{1}^{2}} e^{-\mathbf{j}\left(d_{2} / 2 b_{2}\right) \omega_{2}^{2}}$ results in

$$
\begin{aligned}
& e^{-\mathbf{i}\left(d_{1} / 2 b_{1}\right) \omega_{1}^{2}} L_{A_{1}, A_{2}}^{\mathbb{\boxplus}}\{f\}(\boldsymbol{\omega}) e^{-\mathbf{j}\left(d_{2} / 2 b_{2}\right) \omega_{2}^{2}}=\frac{e^{-\mathbf{i}(\pi / 4)}}{\sqrt{2 \pi b_{1}}} \\
& \cdot \int_{\mathbb{R}^{2}} e^{-\mathbf{i} x_{1}\left(\omega_{1} / b_{1}\right)} \tilde{f}(\mathbf{x}) e^{-\mathbf{j} x_{2}\left(\omega_{2} / b_{2}\right)} d \mathbf{x} \frac{e^{-\mathbf{j}(\pi / 4)}}{\sqrt{2 \pi b_{2}}}=\frac{1}{\sqrt{2 \pi}} \\
& \cdot \int_{\mathbb{R}^{2}} e^{-\mathbf{i} x_{1}\left(\omega_{1} / b_{1}\right)} \frac{e^{-\mathbf{i}(\pi / 4)}}{\sqrt{b_{1}}} \tilde{f}(\mathbf{x}) \frac{e^{-\mathbf{i}(\pi / 4)}}{\sqrt{b_{2}}} e^{-\mathbf{j} x_{2}\left(\omega_{2} / b_{2}\right)} d \mathbf{x} \\
& \cdot \frac{1}{\sqrt{2 \pi}}=\frac{1}{\sqrt{(2 \pi)^{2}}} \\
& \cdot \int_{\mathbb{R}^{2}} e^{-\mathbf{i} x_{1}\left(\omega_{1} / b_{1}\right)} g_{f}(\mathbf{x}) e^{-\mathbf{j} x_{2}\left(\omega_{2} / b_{2}\right)} d \mathbf{x}=\mathscr{F}_{q}\left\{g_{f}\right\} \\
& \cdot\left(\frac{\boldsymbol{\omega}}{\mathbf{b}}\right) \cdot
\end{aligned}
$$

This is the desired result.

Theorem 6. If $f \in L^{1}\left(\mathbb{R}^{2} ; \mathbb{H}\right)$ and $L_{A_{1}, A_{2}}^{\mathbb{H}}\{f\} \in L^{1}\left(\mathbb{R}^{2} ; \mathbb{H}\right)$, then the inverse transform of the $Q L C T$ can be derived from that of the QFT.

Proof. Indeed, we have

$$
\begin{aligned}
g_{f}(\mathbf{x})=\frac{1}{\sqrt{(2 \pi)^{2}}} \int_{\mathbb{R}^{2}} e^{\mathbf{i} x_{1} \omega_{1}} \mathscr{F}_{q}\left\{g_{f}\right\}(\boldsymbol{\omega}) e^{\mathbf{j} x_{2} \omega_{2}} d \boldsymbol{\omega} \\
=\frac{1}{\sqrt{(2 \pi)^{2}}} \int_{\mathbb{R}^{2}} e^{\mathbf{i} x_{1} \omega_{1} \widetilde{\mathscr{F}}(\mathbf{b} \boldsymbol{\omega}) e^{\mathbf{j} x_{2} \omega_{2}} d \boldsymbol{\omega}} \\
=\frac{1}{b_{1} b_{2} \sqrt{(2 \pi)^{2}}} \int_{\mathbb{R}^{2}} e^{\mathbf{i} x_{1}\left(\omega_{1} / b_{1}\right)} \widetilde{\mathscr{F}}(\boldsymbol{\omega}) e^{\mathbf{j} x_{2}\left(\omega_{2} / b_{2}\right)} d \boldsymbol{\omega} \\
=\frac{1}{b_{1} b_{2} \sqrt{(2 \pi)^{2}}} \int_{\mathbb{R}^{2}} e^{\mathbf{i} x_{1}\left(\omega_{1} / b_{1}\right)} e^{-\mathbf{i}\left(d_{1} / 2 b_{1}\right) \omega_{1}^{2}} L_{A_{1}, A_{2}}^{\boxplus-}\{f\} \\
\cdot(\boldsymbol{\omega}) e^{-\mathbf{j}\left(d_{2} / 2 b_{2}\right) \omega_{2}^{2}} e^{\mathbf{j} x_{2}\left(\omega_{2} / b_{2}\right)} d \boldsymbol{\omega} .
\end{aligned}
$$

It means that

$$
\begin{aligned}
& \frac{e^{-\mathbf{i}(\pi / 4)}}{\sqrt{b_{1}}} e^{\mathbf{i}\left(a_{1} / 2 b_{1}\right) x_{1}^{2}} f(\mathbf{x}) e^{\mathbf{j}\left(a_{2} / 2 b_{2}\right) x_{2}^{2}} \frac{e^{-\mathbf{j}(\pi / 4)}}{\sqrt{b_{2}}} \\
& =\frac{1}{b_{1} b_{2} \sqrt{(2 \pi)^{2}}} \int_{\mathbb{R}^{2}} e^{\mathbf{i} x_{1}\left(\omega_{1} / b_{1}\right)} e^{-\mathbf{i}\left(d_{1} / 2 b_{1}\right) \omega_{1}^{2}} L_{A_{1}, A_{2}}^{\boxplus}\{f\} \\
& \cdot(\boldsymbol{\omega}) e^{-\mathbf{j}\left(d_{2} / 2 b_{2}\right) \omega_{2}^{2}} e^{\mathbf{j} x_{2}\left(\omega_{2} / b_{2}\right)} d \boldsymbol{\omega} .
\end{aligned}
$$


Or, equivalently,

$$
\begin{aligned}
& f(\mathbf{x}) \\
& =\int_{\mathbb{R}^{2}} \frac{1}{\sqrt{2 \pi b_{1}}} e^{-\mathbf{i}\left(a_{1} / 2 b_{1}\right) x_{1}^{2}} e^{\mathbf{i} x_{1}\left(\omega_{1} / b_{1}\right)} e^{-\mathbf{i}\left(d_{1} / 2 b_{1}\right) \omega_{1}^{2}} e^{\mathbf{i}(\pi / 4)} L_{A_{1}, A_{2}}^{\mathbb{N}}\{f\} \\
& \cdot(\boldsymbol{\omega}) \frac{1}{\sqrt{2 \pi b_{2}}} e^{-\mathbf{j}\left(a_{2} / 2 b_{2}\right) x_{2}^{2}} e^{\mathbf{j} x_{2}\left(\omega_{2} / b_{2}\right)} e^{-\mathbf{j}\left(d_{2} / 2 b_{2}\right) \omega_{2}^{2}} e^{-\mathbf{i}(\pi / 4)} d \boldsymbol{\omega} \\
& =\int_{\mathbb{R}^{2}} \overline{K_{A_{1}}\left(x_{1}, \omega_{1}\right)} L_{A_{1}, A_{2}}^{\mathbb{H}}\{f\}(\boldsymbol{\omega}) \overline{K_{A_{2}}\left(x_{2}, \omega_{2}\right)} d \boldsymbol{\omega},
\end{aligned}
$$

which is inverse transform of the QLCT. This proves the theorem.

In following we give an alternative proof of Parseval formula for the QLCT (cf. [8]).

Theorem 7 (QLCT Parseval). Two quaternion functions $f, h \in L^{1}\left(\mathbb{R}^{2} ; \mathbb{U}\right) \cap L^{2}\left(\mathbb{R}^{2} ; \mathbb{H}\right)$ are related to their QLCT via the Parseval formula, given as

$$
\langle f, h\rangle_{L^{2}\left(\mathbb{R}^{2} ; \mathbb{W}\right)}=\left\langle L_{A_{1}, A_{2}}^{\mathbb{Q}}\{f\}, L_{A_{1}, A_{2}}^{\mathbb{Q}}\{h\}\right\rangle_{L^{2}\left(\mathbb{R}^{2} ; \mathbb{G}\right)} .
$$

For $f=h$, one has

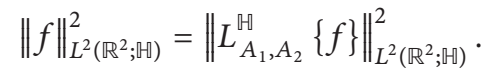

Proof. From Parseval formula (15), it follows that

$$
\begin{aligned}
& \left\langle g_{f}, g_{h}\right\rangle=\left\langle\mathscr{F}_{q}\left\{g_{f}\right\}, \mathscr{F}_{q}\left\{g_{h}\right\}\right\rangle \\
& =\operatorname{Sc} \int_{\mathbb{R}^{2}} \mathscr{F}_{q}\left\{g_{f}\right\}(\boldsymbol{\omega}) \overline{\mathscr{F}_{q}\left\{g_{h}\right\}(\boldsymbol{\omega})} d \boldsymbol{\omega} \\
& =\frac{1}{\left|b_{1} b_{2}\right|} \mathrm{Sc} \int_{\mathbb{R}^{2}} \mathscr{F}_{q}\left\{g_{f}\right\}\left(\frac{\boldsymbol{\omega}}{\mathbf{b}}\right) \overline{\mathscr{F}_{q}\left\{g_{h}\right\}\left(\frac{\boldsymbol{\omega}}{\mathbf{b}}\right)} d \boldsymbol{\omega} .
\end{aligned}
$$

Applying the cyclic multiplication symmetry, we get

$$
\begin{aligned}
& \left\langle g_{f}, g_{h}\right\rangle=\frac{1}{\left|b_{1} b_{2}\right|} \operatorname{Sc} \int_{\mathbb{R}^{2}} e^{-\mathbf{i}\left(d_{1} / 2 b_{1}\right) \omega_{1}^{2}} L_{A_{1}, A_{2}}^{\mathbb{D}}\{f\}(\boldsymbol{\omega}) \\
& \cdot e^{-\mathbf{j}\left(d_{2} / 2 b_{2}\right) \omega_{2}^{2}} \overline{e^{-\mathbf{i}\left(d_{1} / 2 b_{1}\right) \omega_{1}^{2}} L_{A_{1}, A_{2}}^{\mathbb{N}}\{h\}(\boldsymbol{\omega}) e^{-\mathbf{j}\left(d_{2} / 2 b_{2}\right) \omega_{2}^{2}}} d \boldsymbol{\omega} \\
& =\frac{1}{\left|b_{1} b_{2}\right|} \operatorname{Sc} \int_{\mathbb{R}^{2}} e^{-\mathbf{i}\left(d_{1} / 2 b_{1}\right) \omega_{1}^{2}} L_{A_{1}, A_{2}}^{\mathbb{1}}\{f\}(\boldsymbol{\omega}) \\
& \cdot e^{-\mathbf{j}\left(d_{2} / 2 b_{2}\right) \omega_{2}^{2}} e^{-\mathbf{j}\left(d_{2} / 2 b_{2}\right) \omega_{1}^{2}} \overline{L_{A_{1}, A_{2}}^{\mathbb{U}}\{h\}(\boldsymbol{\omega})} e^{-\mathbf{i}\left(d_{1} / 2 b_{1}\right) \omega_{1}^{2}} d \boldsymbol{\omega} \\
& =\frac{1}{\left|b_{1} b_{2}\right|} \operatorname{Sc} \int_{\mathbb{R}^{2}} L_{A_{1}, A_{2}}^{\boxplus}\{f\}(\boldsymbol{\omega}) \overline{L_{A_{1}, A_{2}}^{\mathbb{U}}\{h\}(\boldsymbol{\omega})} d \boldsymbol{\omega} .
\end{aligned}
$$

On the other hand,

$$
\begin{aligned}
& \left\langle g_{f}, g_{h}\right\rangle=\operatorname{Sc} \int_{\mathbb{R}^{2}} g_{f}(\mathbf{x}) \overline{g_{h}(\mathbf{x})} d \mathbf{x} \\
& =\operatorname{Sc} \int_{\mathbb{R}^{2}} \frac{e^{-\mathbf{i}(\pi / 4)}}{\sqrt{b_{1}}} \tilde{f}(\mathbf{x}) \\
& \cdot \frac{e^{-\mathbf{j}(\pi / 4)}}{\sqrt{b_{2}}} \frac{e^{-\mathbf{i}(\pi / 4)}}{\sqrt{b_{1}}} \tilde{h}(\mathbf{x}) \frac{e^{-\mathbf{j}(\pi / 4)}}{\sqrt{b_{2}}} d \mathbf{x} \\
& =\frac{1}{\left|b_{1} b_{2}\right|} \operatorname{Sc} \int_{\mathbb{R}^{2}} \tilde{f}(\mathbf{x}) \overline{\widetilde{h}(\mathbf{x})} d \mathbf{x} \\
& =\frac{1}{\left|b_{1} b_{2}\right|} \operatorname{Sc} \int_{\mathbb{R}^{2}} e^{\mathbf{i}\left(a_{1} / 2 b_{1}\right) x_{1}^{2}} f(\mathbf{x}) \\
& \cdot e^{\mathbf{j}\left(a_{2} / 2 b_{2}\right) x_{2}^{2}} e^{\mathbf{i}\left(a_{1} / 2 b_{1}\right) x_{1}^{2} h(\mathbf{x}) e^{\mathbf{j}\left(a_{2} / 2 b_{2}\right) x_{2}^{2}}} d \mathbf{x} \\
& =\frac{1}{\left|b_{1} b_{2}\right|} \operatorname{Sc} \int_{\mathbb{R}^{2}} f(\mathbf{x}) \overline{h(\mathbf{x})} d \mathbf{x} .
\end{aligned}
$$

The proof is complete.

It is interesting to describe the relationship between the QLCT and QFT as shown in the following example.

Example 8. Let us now compute the QLCT of the twodimensional Gaussian function $f(\mathbf{x})=e^{-\left(k_{1} x_{1}^{2}+k_{2} x_{2}^{2}\right)}$ with $k_{1}, k_{2}>0$.

From the definition of QLCT (17), we easily obtain

$$
\begin{aligned}
& L_{A_{1}, A_{2}}^{\mathbb{H}}\{f\}(\boldsymbol{\omega})=\frac{1}{\sqrt{2 \pi b_{1}}} \\
& \cdot \int_{\mathbb{R}^{2}} e^{\mathbf{i}(1 / 2)\left(\left(a_{1} / b_{1}\right) x_{1}^{2}-\left(2 / b_{1}\right) x_{1} \omega+\left(d_{1} / b_{1}\right) \omega^{2}-(\pi / 2)\right)} f(\mathbf{x}) \\
& \cdot e^{\mathbf{j}(1 / 2)\left(\left(a_{2} / b_{2}\right) x_{2}^{2}-\left(2 / b_{2}\right) x_{2} \omega+\left(d_{2} / b_{2}\right) \omega^{2}-(\pi / 2)\right)} \frac{1}{\sqrt{2 \pi b_{2}}} d \mathbf{x} \\
& =\frac{1}{\sqrt{2 \pi b_{1}}} \\
& \cdot \int_{\mathbb{R}} e^{-k_{1} x_{1}^{2}} e^{\mathbf{i}(1 / 2)\left(\left(a_{1} / b_{1}\right) x_{1}^{2}-\left(2 / b_{1}\right) x_{1} \omega+\left(d_{1} / b_{1}\right) \omega^{2}-(\pi / 2)\right)} d x_{1} \\
& \cdot \frac{1}{\sqrt{2 \pi b_{2}}} \\
& \cdot \int_{\mathbb{R}} e^{-k_{2} x_{2}^{2}} e^{\mathbf{j}(1 / 2)\left(\left(a_{2} / b_{2}\right) x_{2}^{2}-\left(2 / b_{2}\right) x_{2} \omega_{2}+\left(d_{2} / b_{2}\right) \omega^{2}-(\pi / 2)\right)} d x_{2} \\
& =\frac{1}{\sqrt{2 \pi b_{1}}} \\
& \cdot e^{\mathbf{i}(1 / 2)\left(d_{1} / b_{1}\right) \omega^{2}} \int_{\mathbb{R}} e^{-\left(1 / 2 b_{1}\right)\left(2 k_{1} b_{1}-\mathbf{i} a_{1}\right) x_{1}^{2}} e^{-\mathbf{i}\left(\omega / b_{1}\right) x_{1}} e^{-\mathbf{i}(\pi / 2)} d x_{1} \\
& \cdot \frac{1}{\sqrt{2 \pi b_{2}}} \\
& \cdot e^{\mathbf{j}(1 / 2)\left(d_{2} / b_{2}\right) \omega^{2}} \int_{\mathbb{R}} e^{-\left(1 / 2 b_{2}\right)\left(2 k_{2} b_{2}-\mathbf{j} a_{2}\right) x_{2}^{2}} e^{-\mathbf{j}\left(\omega / b_{2}\right) x_{2}} e^{-\mathbf{j}(\pi / 2)} d x_{2} .
\end{aligned}
$$


Using the QFT of the Gaussian function,

$$
\begin{aligned}
\mathscr{F}_{q}\{f\}(\boldsymbol{\omega}) & =\int_{\mathbb{R}^{2}} e^{-\mathbf{i} \omega_{1} x_{1}} e^{-\left(k_{1} x_{1}^{2}+k_{2} x_{2}^{2}\right)} e^{-\mathbf{j} \omega_{2} x_{2}} d \mathbf{x} \\
& =\frac{\pi}{\sqrt{k_{1} k_{2}}} e^{-\left(\omega_{1}^{2} / 4 k_{1}+\omega_{2}^{2} / 4 k_{2}\right)} .
\end{aligned}
$$

We immediately obtain

$$
\begin{aligned}
& L_{A_{1}, A_{2}}^{\boxplus !}\{f\}(\boldsymbol{\omega})=\frac{1}{\sqrt{2 \pi b_{1} \mathbf{i}}} \\
& \cdot e^{\mathbf{i}(1 / 2)\left(d_{1} / b_{1}\right) \omega_{1}^{2}} \sqrt{\frac{2 \pi b_{1}}{\left(2 k_{1} b_{1}-\mathbf{i} a_{1}\right)}} e^{-\omega_{1}^{2} / 2 b_{1}\left(2 k_{1} b_{1}-\mathbf{i} a_{1}\right)} \\
& \cdot \frac{1}{\sqrt{2 \pi b_{1}}} \\
& \cdot e^{\mathbf{j}(1 / 2)\left(d_{2} / b_{2}\right) \omega_{2}^{2}} \sqrt{\frac{2 \pi b_{2}}{\left(2 k_{2} b_{2}-\mathbf{j} a_{2}\right)}} e^{-\omega_{2}^{2} / 2 b_{2}\left(2 k_{2} b_{2}-\mathbf{j} a_{2}\right)} \\
& =\frac{1}{\sqrt{a_{1}+2 k_{1} b_{1}}} e^{\left(\omega_{1}^{2} / 2\right)\left(\left(c_{1}+2 k_{1} \mathbf{i} d_{1}\right) /\left(2 k_{1} b_{1}-\mathbf{i} a_{1}\right)\right)} \\
& \cdot \frac{1}{\sqrt{a_{2}+2 k_{2} b_{2}}} e^{\left(\omega_{2}^{2} / 2\right)\left(\left(c_{2}+2 k_{2} \mathbf{j} d_{2}\right) /\left(2 k_{2} b_{2}-\mathbf{j} a_{2}\right)\right)} .
\end{aligned}
$$

$$
\begin{aligned}
& L_{A_{1}, A_{2}}^{\boxplus \Perp}\{f\}(\boldsymbol{\omega})=\int_{\mathbb{R}^{2}} \frac{1}{\sqrt{2 \pi b_{1}}} e^{\mathbf{i}(1 / 2)\left(\left(a_{1} / b_{1}\right) x_{1}^{2}-\left(2 / b_{1}\right) x_{1} \omega_{1}+\left(d_{1} / b_{1}\right) \omega_{1}^{2}-\pi / 2\right)} f(\mathbf{x}) \frac{1}{\sqrt{2 \pi b_{2}}} e^{\mathbf{j}(1 / 2)\left(\left(a_{2} / b_{2}\right) x_{2}^{2}-\left(2 / b_{2}\right) x_{2} \omega_{2}+\left(d_{2} / b_{2}\right) \omega_{2}^{2}-\pi / 2\right)} d \mathbf{x} \\
& =-\int_{\mathbb{R}^{2}} \frac{1}{\sqrt{2 \pi b_{1}}} e^{\mathbf{i}(1 / 2)\left(\left(a_{1} / b_{1}\right) x_{1}^{2}-\left(2 / b_{1}\right)\left(x_{1}+b_{1} \pi / \omega_{1}\right) \omega_{1}+\left(d_{1} / b_{1}\right) \omega_{1}^{2}-\pi / 2\right)} f(\mathbf{x}) \frac{1}{\sqrt{2 \pi b_{2}}} e^{\mathbf{j}(1 / 2)\left(\left(a_{2} / b_{2}\right) x_{2}^{2}-\left(2 / b_{2}\right) x_{2} \omega_{2}+\left(d_{2} / b_{2}\right) \omega_{2}^{2}-\pi / 2\right)} d \mathbf{x} .
\end{aligned}
$$

Now applying (38) gives

Proof. It is not difficult to see that

$$
\begin{aligned}
& e^{-\mathbf{i}\left(\omega_{1} x_{1} / b_{1}\right)}=-e^{-\mathbf{i}\left(\omega_{1} / b_{1}\right)\left(x_{1}+b_{1} \pi / \omega_{1}\right)}, \\
& e^{-\mathbf{j}\left(\omega_{2} x_{2} / b_{2}\right)}=-e^{-\mathbf{j}\left(\omega_{2} / b_{2}\right)\left(x_{2}+b_{2} \pi / \omega_{2}\right)} .
\end{aligned}
$$

In this section we present useful properties of the QLCT in detail. We see that the results are generalizations of the asymptotic behavior of the QLCT. In the following, we shall provide a different proof of the results using the QLCT kernel

3.1. Asymptotic Behavior of the QLCT. Like the classical Fourier transform, the Riemann-Lebesgue lemma is also

Theorem 9 (Riemann-Lebesgue lemma). Suppose that $f \in$

$$
\begin{aligned}
& \lim _{\left|\omega_{1}\right| \rightarrow \infty}\left|L_{A_{1}, A_{2}}^{\mathbb{Q}}\{f\}(\boldsymbol{\omega})\right|=0, \\
& \lim _{\left|\omega_{2}\right| \rightarrow \infty}\left|L_{A_{1}, A_{2}}^{\mathbb{\sharp}}\{f\}(\boldsymbol{\omega})\right|=0 .
\end{aligned}
$$

Therefore, by making the change of variable $x_{1}+b_{1} \pi / \omega_{1}=t_{1}$ in the above identity, we immediately obtain

$$
\begin{aligned}
& L_{A_{1}, A_{2}}^{\mathbb{Q}}\{f\}(\boldsymbol{\omega})=-\int_{\mathbb{R}^{2}} \frac{1}{\sqrt{2 \pi b_{1}}} e^{\mathbf{i}(1 / 2)\left(\left(a_{1} / b_{1}\right)\left(t_{1}-b_{1} \pi / \omega_{1}\right)^{2}-\left(2 / b_{1}\right) t_{1} \omega_{1}+\left(d_{1} / b_{1}\right) \omega_{1}^{2}-\pi / 2\right)} f\left(t_{1}-\frac{b_{1} \pi}{\omega_{1}}, t_{2}\right) \\
& \cdot \frac{1}{\sqrt{2 \pi b_{2}}} e^{\mathbf{j}(1 / 2)\left(\left(a_{2} / b_{2}\right) t_{2}^{2}-\left(2 / b_{1}\right) t_{2} \omega_{1}+\left(d_{1} / b_{2}\right) \omega_{2}^{2}-\pi / 2\right)} d \mathbf{t}=\frac{1}{2}\left[\int_{\mathbb{R}^{2}} \frac{1}{\sqrt{2 \pi b_{1}}} e^{\mathbf{i}(1 / 2)\left(\left(a_{1} / b_{1}\right) t_{1}^{2}-\left(2 / b_{1}\right) t_{1} \omega_{1}+\left(d_{1} / b_{1}\right) \omega_{1}^{2}-\pi / 2\right)} f(\mathbf{t})\right. \\
& \cdot \frac{1}{\sqrt{2 \pi b_{2}}} e^{\mathbf{j}(1 / 2)\left(\left(a_{2} / b_{2}\right) t_{2}^{2}-\left(2 / b_{2}\right) t_{2} \omega_{2}+\left(d_{2} / b_{2}\right) \omega_{2}^{2}-\pi / 2\right)} d \mathbf{x} \\
& \left.-\int_{\mathbb{R}^{2}} \frac{1}{\sqrt{2 \pi b_{1}}} e^{\mathbf{i}(1 / 2)\left(\left(a_{1} / b_{1}\right)\left(t_{1}-b_{1} \pi / \omega_{1}\right)^{2}-\left(2 / b_{1}\right) t_{1} \omega_{1}+\left(d_{1} / b_{1}\right) \omega_{1}^{2}-\pi / 2\right)} f\left(t_{1}-\frac{b_{1} \pi}{\omega_{1}}, t_{2}\right) \frac{1}{\sqrt{2 \pi b_{2}}} e^{\mathbf{j}(1 / 2)\left(\left(a_{2} / b_{2}\right) t_{2}^{2}-\left(2 / b_{2}\right) t_{2} \omega_{2}+\left(d_{2} / b_{2}\right) \omega_{2}^{2}-\pi / 2\right)} d \mathbf{t}\right]
\end{aligned}
$$




$$
\begin{aligned}
& =\frac{1}{2}\left[\int_{\mathbb{R}^{2}} \frac{1}{\sqrt{2 \pi b_{1}}} e^{\mathbf{i}(1 / 2)\left(\left(a_{1} / b_{1}\right) t_{1}^{2}-\left(2 / b_{1}\right) t_{1} \omega_{1}+\left(d_{1} / b_{1}\right) \omega_{1}^{2}-\pi / 2\right)}\left(f(\mathbf{t})-e^{\mathbf{i}\left(a_{1} / 2 b_{1}\right)\left(-2 t_{1} b_{1} \pi / \omega_{1}+\left(b_{1} \pi / \omega_{1}\right)^{2}\right)} f\left(t_{1}-\frac{b_{1} \pi}{\omega_{1}}, t_{2}\right)\right)\right. \\
& \left.\cdot \frac{1}{\sqrt{2 \pi b_{2}}} e^{\mathbf{j}(1 / 2)\left(\left(a_{2} / b_{2}\right) t_{2}^{2}-\left(2 / b_{2}\right) t_{2} \omega_{2}+\left(d_{2 s} / b_{2}\right) \omega_{2}^{2}-\pi / 2\right)} d \mathbf{t}\right] .
\end{aligned}
$$

This means that

$$
\begin{aligned}
& \lim _{\left|\omega_{1}\right| \rightarrow \infty}\left|L_{A_{1}, A_{2}}^{\mathbb{⿴}}\{f\}(\boldsymbol{\omega})\right| \leq \frac{1}{\left|4 \pi b_{1} b_{2}\right|} \lim _{\left|\omega_{1}\right| \rightarrow \infty} \int_{\mathbb{R}^{2}} \mid f(\mathbf{t}) \\
& \quad-e^{\mathbf{i}\left(a_{1} / 2 b_{1}\right)\left(-2 t_{1} b_{1} \pi / \omega_{1}+\left(b_{1} \pi / \omega_{1}\right)^{2}\right)} f\left(t_{1}-\frac{b_{1} \pi}{\omega_{1}}, t_{2}\right) \mid d \mathbf{t} \\
& \quad=0 .
\end{aligned}
$$

\section{Similarly we can prove}

$$
\lim _{\left|\omega_{2}\right| \rightarrow \infty}\left|L_{A_{1}, A_{2}}^{\llbracket}\{f\}(\boldsymbol{\omega})\right|=0 .
$$

Theorem 10 (continuity). If $f \in L^{1}\left(\mathbb{R}^{2} ; \mathbb{H}\right)$, then $L_{A_{1}, A_{2}}^{\mathbb{Q}}\{f\}(\boldsymbol{\omega})$ is continuous on $\mathbb{R}^{2}$.

Proof. Simple computations show that

$$
\begin{aligned}
& \left|L_{A_{1}, A_{2}}^{\mathbb{U}}\{f\}(\boldsymbol{\omega}+\mathbf{h})-L_{A_{1}, A_{2}}^{\mathbb{H}}\{f\}(\boldsymbol{\omega})\right|=\mid \int_{\mathbb{R}^{2}} \frac{1}{\sqrt{2 \pi b_{1}}} e^{\mathbf{i}(1 / 2)\left(\left(a_{1} / b_{1}\right)\left(x_{1}+h_{1}\right)^{2}-\left(2 / b_{1}\right)\left(x_{1}+h_{1}\right) \omega_{1}+\left(d_{1} / b_{1}\right) \omega_{1}^{2}-\pi / 2\right)} f(\mathbf{x}) \\
& \cdot \frac{1}{\sqrt{2 \pi b_{2}}} e^{\mathbf{j}(1 / 2)\left(\left(a_{2} / b_{2}\right)\left(x_{2}+h_{2}\right)^{2}-\left(2 / b_{2}\right)\left(x_{2}+h_{2}\right) \omega_{2}+\left(d_{2} / b_{2}\right) \omega_{2}^{2}-\pi / 2\right)} d \mathbf{x}-\frac{1}{\sqrt{2 \pi b_{1}}} e^{\mathbf{i}(1 / 2)\left(\left(a_{1} / b_{1}\right) x_{1}^{2}-\left(2 / b_{1}\right) x_{1} \omega_{1}+\left(d_{1} / b_{1}\right) \omega_{1}^{2}-\pi / 2\right)} f(\mathbf{x}) \frac{1}{\sqrt{2 \pi b_{2}}} \\
& \cdot e^{\mathbf{j}(1 / 2)\left(\left(a_{2} / b_{2}\right) x_{2}^{2}-\left(2 / b_{2}\right) x_{2} \omega_{2}+\left(d_{2} / b_{2}\right) \omega_{2}^{2}-\pi / 2\right)} d \mathbf{x}\left|\leq \int_{\mathbb{R}^{2}}\right| \frac{1}{\sqrt{2 \pi b_{1}}} \\
& \cdot e^{\mathbf{i}(1 / 2)\left(\left(a_{1} / b_{1}\right) x_{1}^{2}-\left(2 / b_{1}\right) x_{1} \omega_{1}+\left(d_{1} / b_{1}\right) \omega_{1}^{2}-\pi / 2\right)}\left(e^{\mathbf{i}(1 / 2)\left(\left(a_{1} / b_{1}\right)\left(2 x_{1} h_{1}+h_{1}^{2}\right)-\left(2 / b_{1}\right) \omega_{1} h_{1}\right)} f(\mathbf{x}) e^{\mathbf{j}(1 / 2)\left(\left(a_{2} / b_{2}\right)\left(2 x_{2} h_{2}+h_{2}^{2}\right)-\left(2 / b_{2}\right) \omega_{2} h_{2}\right)}-f(\mathbf{x})\right) \\
& \cdot \frac{1}{\sqrt{2 \pi b_{2}}} e^{\mathbf{j}(1 / 2)\left(\left(a_{2} / b_{2}\right) x_{2}^{2}-\left(2 / b_{2}\right) x_{2} \omega_{2}+\left(d_{2} / b_{2}\right) \omega_{2}^{2}-\pi / 2\right)} \mid d \mathbf{x}=\frac{1}{\left|2 \pi b_{1} b_{2}\right|} \\
& \cdot \int_{\mathbb{R}^{2}}\left|\left(e^{\mathbf{i}(1 / 2)\left(\left(a_{1} / b_{1}\right)\left(2 x_{1} h_{1}+h_{1}^{2}\right)-\left(2 / b_{1}\right) \omega_{1} h_{1}\right)} f(\mathbf{x}) e^{\mathbf{j}(1 / 2)\left(\left(a_{2} / b_{2}\right)\left(2 x_{2} h_{2}+h_{2}^{2}\right)-\left(2 / b_{2}\right) \omega_{2} h_{2}\right)}-f(\mathbf{x})\right)\right| d \mathbf{x} \leq \frac{1}{\left|\pi b_{1} b_{2}\right|} \int_{\mathbb{R}^{2}}|f(\mathbf{x})| d \mathbf{x} .
\end{aligned}
$$

By the Lebesgue dominated convergence theorem, we may conclude that

$$
\left|L_{A_{1}, A_{2}}^{\mathbb{Q}}\{f\}(\boldsymbol{\omega}+\mathbf{h})-L_{A_{1}, A_{2}}^{\mathbb{\sharp}}\{f\}(\boldsymbol{\omega})\right| \longrightarrow 0
$$

when $\mathbf{h} \rightarrow 0$. This proves that $L_{A_{1}, A_{2}}^{\mathbb{U}}\{f\}(\boldsymbol{\omega})$ is continuous on $\mathbb{R}^{2}$. Again since (43) is independent of $\boldsymbol{\omega}, L_{A_{1}, A_{2}}^{\mathbb{\#}}\{f\}(\boldsymbol{\omega})$ is, in fact, uniformly continuous on $\mathbb{R}^{2}$.

3.2. Useful Properties of the QLCT. Due to the noncommutativity of the kernel of the QLCT, we only have a left linearity property with specific constants

$$
\alpha, \beta \in\left\{q \mid q=q_{0}+\mathbf{i} q_{1}, q_{0}, q_{1} \in \mathbb{R}\right\},
$$

which is

$$
\begin{aligned}
L_{A_{1}, A_{2}}^{\boxplus \boxplus}\{\alpha f+\beta g\}(\boldsymbol{\omega})= & \alpha L_{A_{1}, A_{2}}^{\mathbb{1}}\{f\}(\boldsymbol{\omega}) \\
& +\beta L_{A_{1}, A_{2}}^{\mathbb{Q}}\{g\}(\boldsymbol{\omega}),
\end{aligned}
$$

and a right linearity property with specific constants

$$
\alpha^{\prime}, \beta^{\prime} \in\left\{q \mid q=q_{0}+\mathbf{j} q_{2}, q_{0}, q_{2} \in \mathbb{R}\right\} .
$$

Theorem 11 (shift property). Given a quaternion function $f \in$ $L^{2}\left(\mathbb{R}^{2} ; \mathbb{H}\right)$, let $\tau_{\mathbf{k}} f(\mathbf{x})$ denote the shifted (translated) function defined by $\tau_{\mathbf{k}} f(\mathbf{x})=f(\mathbf{x}-\mathbf{k})$, where $\mathbf{k} \in \mathbb{R}^{2}$. Then one gets

$$
\begin{aligned}
L_{A_{1}, A_{2}}^{\mathbb{Q}}\left\{\tau_{\mathbf{k}} f\right\}(\boldsymbol{\omega})= & e^{-\mathbf{i} a_{1} c_{1} k_{1}^{2} / 2+\mathbf{i} c_{1} k_{1} \omega_{1}} L_{A_{1}, A_{2}}^{\mathbb{Q}}\{f\} \\
& \cdot\left(\omega_{1}-a_{1} k_{1}, \omega_{2}-a_{2} k_{2}\right)
\end{aligned}
$$


Proof. Taking into account the definition of QLCT (17), we get

$$
\begin{aligned}
& L_{A_{1}, A_{2}}^{\mathbb{Q}}\left\{\tau_{\mathbf{k}} f\right\}(\boldsymbol{\omega})=\frac{1}{\sqrt{2 \pi b_{1}}} \\
& \quad \cdot \int_{\mathbb{R}^{2}} e^{\mathbf{i}(1 / 2)\left(\left(a_{1} / b_{1}\right) x_{1}^{2}-\left(2 / b_{1}\right) x_{1} \omega_{1}+\left(d_{1} / b_{1}\right) \omega_{1}^{2}-\pi / 2\right)} f(\mathbf{x}-\mathbf{k})
\end{aligned}
$$

$$
\cdot \frac{1}{\sqrt{2 \pi b_{2}}} e^{\mathbf{j}(1 / 2)\left(\left(a_{2} / b_{2}\right) x_{2}^{2}-\left(2 / b_{2}\right) x_{2} \omega_{2}+\left(d_{2} / b_{2}\right) \omega_{2}^{2}-\pi / 2\right)} d \mathbf{x} \text {. }
$$

By making the change of a variable $\mathbf{x}-\mathbf{k}=\mathbf{m}$, we easily obtain

$$
\begin{aligned}
& L_{A_{1}, A_{2}}^{\mathbb{Q}}\left\{\tau_{\mathbf{k}} f\right\}(\boldsymbol{\omega})=\int_{\mathbb{R}^{2}} \frac{1}{\sqrt{2 \pi b_{1}}} e^{\mathbf{i}(1 / 2)\left(\left(a_{1} / b_{1}\right)\left(m_{1}+k_{1}\right)^{2}-\left(2 / b_{1}\right)\left(m_{1}+k_{1}\right) \omega_{1}+\left(d_{1} / b_{1}\right) \omega_{1}^{2}-\pi / 2\right)} f(\mathbf{m}) \\
& \cdot \frac{1}{\sqrt{2 \pi b_{2}}} e^{\mathbf{j}(1 / 2)\left(\left(a_{2} / b_{2}\right)\left(m_{2}+k_{2}\right)^{2}-\left(2 / b_{2}\right)\left(m_{2}+k_{2}\right) \omega_{2}+\left(d_{2} / b_{2}\right) \omega_{2}^{2}-\pi / 2\right)} d \mathbf{m}=\frac{1}{\sqrt{2 \pi b_{1}}} \\
& \cdot \int_{\mathbb{R}^{2}} e^{\mathbf{i}(1 / 2)\left(\left(a_{1} / b_{1}\right) m_{1}^{2}-\left(2 / b_{1}\right) m_{1}\left(\omega_{1}-k_{1} a_{1}\right)+\left(d_{1} / b_{1}\right) \omega_{1}^{2}-\pi / 2\right)} e^{\mathbf{i}\left((1 / 2)\left(a_{1} / b_{1}\right) k_{1}^{2}\right)} e^{\mathbf{i}\left(-(1 / 2)\left(2 k_{1} \omega_{1} / b_{1}\right)\right)} f(\mathbf{m}) \\
& \cdot \frac{1}{\sqrt{2 \pi b_{2}}} e^{\mathbf{j}(1 / 2)\left(\left(a_{2} / b_{2}\right) m_{2}^{2}-\left(2 / b_{2}\right) m_{2}\left(\omega_{2}-k_{2} a_{2}\right)+\left(d_{2} / b_{2}\right) \omega_{2}^{2}-\pi / 2\right)} e^{\mathbf{j}\left((1 / 2)\left(a_{2} / b_{2}\right) k_{2}^{2}\right)} e^{\mathbf{j}\left(-(1 / 2)\left(2 k_{2} \omega_{2} / b_{2}\right)\right)} d \mathbf{m} .
\end{aligned}
$$

Therefore, we further get

$$
\begin{aligned}
& L_{A_{1}, A_{2}}^{\mathbb{H}}\left\{\tau_{\mathbf{k}} f\right\}(\boldsymbol{\omega})=\frac{1}{\sqrt{2 \pi b_{1}}} \int_{\mathbb{R}^{2}} e^{\mathbf{i}(1 / 2)\left(\left(a_{1} / b_{1}\right) m_{1}^{2}-\left(2 / b_{1}\right) m_{1}\left(\omega_{1}-k_{1} a_{1}\right)+\left(d_{1} / b_{1}\right)\left(\omega_{1}-k_{1} a_{1}+k_{1} a_{1}\right)^{2}-\pi / 2\right)} e^{\mathbf{i}\left((1 / 2)\left(a_{1} / b_{1}\right) k_{1}^{2}\right)} e^{\mathbf{i}\left(-(1 / 2)\left(2 k_{1} \omega_{1} / b_{1}\right)\right)} f(\mathbf{m}) \\
& \cdot \sqrt{2 \pi b_{2}} e^{\mathbf{j}(1 / 2)\left(\left(a_{2} / b_{2}\right) m_{2}^{2}-\left(2 / b_{2}\right) m_{2}\left(\omega_{2}-k_{2} a_{2}\right)+\left(d_{2} / b_{2}\right)\left(\omega_{2}-k_{2} a_{2}+k_{2} a_{2}\right)^{2}-\pi / 2\right)} e^{\mathbf{j}\left((1 / 2)\left(a_{1} / b_{1}\right) k_{1}^{2}\right)} e^{\mathbf{j}\left(-(1 / 2)\left(2 k_{2} \omega_{2} / b_{2}\right)\right)} d \mathbf{m} \\
& =e^{\mathbf{i}(1 / 2)\left(d_{1} / b_{1}\right)\left(2\left(\omega_{1}-k_{1} a_{1}\right) k_{1} a_{1}+\left(k_{1} a_{1}\right)^{2}\right)} e^{\mathbf{i}\left((1 / 2)\left(a_{1} / b_{1}\right) k_{1}^{2}\right)} e^{\mathbf{i}\left(-(1 / 2)\left(2 k_{1} \omega_{1} / b_{1}\right)\right)} \int_{\mathbb{R}^{2}} \frac{1}{\sqrt{2 \pi b_{1}}} e^{\mathbf{i}(1 / 2)\left(\left(a_{1} / b_{1}\right) m_{1}^{2}-\left(2 / b_{1}\right) m_{1}\left(\omega_{1}-k_{1} a_{1}\right)+\left(d_{1} / b_{1}\right)\left(\omega_{1}-k_{1} a_{1}\right)^{2}\right)} f(\mathbf{m}) \\
& \cdot \frac{1}{\sqrt{2 \pi b_{2}}} e^{\mathbf{j}(1 / 2)\left(\left(a_{2} / b_{2}\right) m_{2}^{2}-\left(2 / b_{2}\right) m_{2}\left(\omega_{2}-k_{2} a_{2}\right)+\left(d_{2} / b_{2}\right)\left(\omega_{2}-k_{2} a_{2}\right)^{2}\right)} d \mathbf{m} e^{\mathbf{j}(1 / 2)\left(d_{2} / b_{2}\right)\left(2\left(\omega_{2}-k_{2} a_{2}\right) k_{2} a_{2}+\left(k_{2} a_{2}\right)^{2}\right)} e^{\mathbf{j}\left((1 / 2)\left(a_{2} / b_{2}\right) k_{2}^{2}\right)} e^{\mathbf{j}\left(-(1 / 2)\left(2 k_{2} \omega_{2} / b_{2}\right)\right)} .
\end{aligned}
$$

Applying the definition of the QLCT (17), the above expression can be rewritten in the form

$$
\begin{aligned}
& L_{A_{1}, A_{2}}^{\mathbb{U}}\left\{\tau_{\mathbf{k}} f\right\}(\boldsymbol{\omega})=e^{\mathbf{i}(1 / 2)\left(d_{1} / b_{1}\right)\left(2\left(\omega_{1}-k_{1} a_{1}\right) k_{1} a_{1}+\left(k_{1} a_{1}\right)^{2}\right)} e^{\mathbf{i}\left((1 / 2)\left(a_{1} / b_{1}\right) k_{1}^{2}\right)} e^{\mathbf{i}\left(-(1 / 2)\left(2 k_{1} \omega_{1} / b_{1}\right)\right)} L_{A_{1}, A_{2}}^{\boxplus \Perp}\{f\}\left(\omega_{1}-a_{1} k_{1}, \omega_{2}-a_{2} k_{2}\right) \\
& \cdot e^{\mathbf{j}(1 / 2)\left(d_{2} / b_{2}\right)\left(2\left(\omega_{1}-k_{2} a_{2}\right) k_{2} a_{2}+\left(k_{2} a_{2}\right)^{2}\right)} e^{\mathbf{j}\left((1 / 2)\left(a_{2} / b_{2}\right) k_{2}^{2}\right)} e^{\mathbf{j}\left(-(1 / 2)\left(2 k_{2} \omega_{2} / b_{2}\right)\right)}
\end{aligned}
$$

We notice that

$$
\begin{aligned}
& e^{\mathbf{i}(1 / 2)\left(d_{1} / b_{1}\right)\left(2\left(\omega_{1}-k_{1} a_{1}\right) k_{1} a_{1}+\left(k_{1} a_{1}\right)^{2}\right)} e^{\mathbf{i}\left((1 / 2)\left(a_{1} / b_{1}\right) k_{1}^{2}\right)} e^{\mathbf{i}\left(-(1 / 2)\left(2 k_{1} \omega_{1} / b_{1}\right)\right)} \\
& =e^{\mathbf{i} k_{1} \omega_{1}\left(d_{1} a_{1} / b_{1}-1 / b_{1}\right)} e^{-\mathbf{i}(1 / 2) k_{1}^{2} a_{1}\left(d_{1} a_{1} / b_{1}-1 / b_{1}\right)}, \\
& e^{\mathbf{j}(1 / 2)\left(d_{2} / b_{2}\right)\left(2\left(\omega_{2}-k_{2} a_{2}\right) k_{2} a_{2}+\left(k_{2} a_{2}\right)^{2}\right)} e^{\mathbf{j}\left((1 / 2)\left(a_{2} / b_{2}\right) k_{2}^{2}\right)} e^{\mathbf{j}\left(-(1 / 2)\left(2 k_{2} \omega_{2} / b_{2}\right)\right)} \\
& =e^{\mathbf{j} k_{2} \omega_{2}\left(d_{2} a_{2} / b_{2}-1 / b_{2}\right)} e^{-\mathbf{j}(1 / 2) k_{2}^{2} a_{2}\left(d_{2} a_{2} / b_{2}-1 / b_{2}\right)} .
\end{aligned}
$$

$$
\begin{aligned}
& e^{\mathbf{i}(1 / 2)\left(d_{1} / b_{1}\right)\left(2\left(\omega_{1}-k_{1} a_{1}\right) k_{1} a_{1}+\left(k_{1} a_{1}\right)^{2}\right)} e^{\mathbf{i}\left((1 / 2)\left(a_{1} / b_{1}\right) k_{1}^{2}\right)} e^{\mathbf{i}\left(-(1 / 2)\left(2 k_{1} \omega_{1} / b_{1}\right)\right)} \\
& =e^{\mathbf{i} k_{1} \omega_{1} c_{1}} e^{-\mathbf{i}\left(a_{1} k_{1}^{2} / 2\right) c_{1}} .
\end{aligned}
$$


By the above equalities, we finally arrive at

$$
\begin{aligned}
L_{A_{1}, A_{2}}^{\mathbb{Q}}\left\{\tau_{\mathbf{k}} f\right\}(\boldsymbol{\omega})= & e^{\mathbf{i} k_{1} \omega_{1} c_{1}} e^{-\mathbf{i}\left(a_{1} k_{1}^{2} / 2\right) c_{1}} L_{A_{1}, A_{2}}^{\mathbb{D}}\{f\} \\
& \cdot\left(\omega_{1}-a_{1} k_{1}, \omega_{2}-a_{2} k_{2}\right) \\
& \cdot e^{\mathrm{j} k_{2} \omega_{2} c_{2}} e^{-\mathbf{j}\left(a_{2} k_{2}^{2} / 2\right) c_{2}} .
\end{aligned}
$$

This completes the proof of theorem.

Next, we are concerned with the behavior of the QLCT under modulation.

Theorem 12 (modulation property). Let $\mathbb{M}_{\omega_{0}} f$ be modulation operator defined by $\mathbb{M}_{\boldsymbol{\omega}_{0}} f(\mathbf{x})=e^{\mathbf{i} x_{1} u_{0}} f(\mathbf{x}) e^{\mathbf{j} x_{2} v_{0}}$ with $\boldsymbol{\omega}_{0}=$ $u_{0} \mathbf{e}_{1}+v_{0} \mathbf{e}_{2}$. Then

$$
\begin{aligned}
& L_{A_{1}, A_{2}}^{\mathbb{Q}}\left\{\mathbb{M}_{\boldsymbol{\omega}_{0}} f\right\}(\boldsymbol{\omega})=L_{A_{1}, A_{2}}^{\mathbb{Q}}\left\{e^{\mathbf{i} x_{1} u_{0}} f(\mathbf{x}) e^{\mathbf{j} x_{2} v_{0}}\right\}(\boldsymbol{\omega}) \\
& =e^{-\mathbf{i} b_{1} d_{1} u_{0}^{2} / 2+\mathbf{i} d_{1} u_{0} \omega_{1}} L_{A_{1}, A_{2}}^{\mathbb{Q}}\{f\} \\
& \cdot\left(\omega_{1}-u_{0} b_{1}, \omega_{2}-v_{0} b_{2}\right) e^{-\mathbf{j} b_{2} d_{2} v_{0}^{2} / 2+\mathbf{j} d_{2} v_{0} \omega_{2}} \text {. }
\end{aligned}
$$

$$
\begin{aligned}
& L_{A_{1}, A_{2}}^{\mathbb{N}}\left\{\mathbb{M}_{\boldsymbol{\omega}_{0}} f\right\}(\boldsymbol{\omega})=\frac{1}{\sqrt{2 \pi b_{1}}} \int_{\mathbb{R}^{2}} e^{\mathbf{i}(1 / 2)\left(\left(a_{1} / b_{1}\right) x_{1}^{2}-\left(2 / b_{1}\right) x_{1}\left(\omega_{1}-u_{0} b_{1}\right)+\left(d_{1} / b_{1}\right)\left(\left(\omega_{1}-u_{0} b_{1}\right)+u_{0} b_{1}\right)^{2}-\pi / 2\right)} f(\mathbf{x}) \\
& \cdot \frac{1}{\sqrt{2 \pi b_{2}}} e^{\mathbf{j}(1 / 2)\left(\left(a_{2} / b_{2}\right) x_{2}^{2}-\left(2 / b_{2}\right) x_{2}\left(\omega_{2}-v_{0} b_{2}\right)+\left(d_{2} / b_{2}\right)\left(\left(\omega_{2}-v_{0} b_{2}\right)+v_{0} b_{2}\right)^{2}-\pi / 2\right)} d \mathbf{x}=\frac{1}{\sqrt{2 \pi b_{1}}} \\
& \cdot \int_{\mathbb{R}^{2}} e^{\mathbf{i}(1 / 2)\left(\left(a_{1} / b_{1}\right) x_{1}^{2}-\left(2 / b_{1}\right) x_{1}\left(\omega_{1}-u_{0} b_{1}\right)+\left(d_{1} / b_{1}\right)\left(\left(\omega_{1}-u_{0} b_{1}\right)^{2}+2\left(\omega_{1}-u_{0} b_{1}\right) u_{0} b_{1}+u_{0}^{2} b_{1}^{2}\right)-\pi / 2\right)} f(\mathbf{x}) \frac{1}{\sqrt{2 \pi b_{2}}} \\
& \cdot e^{\mathbf{j}(1 / 2)\left(\left(a_{2} / b_{2}\right) x_{2}^{2}-\left(2 / b_{2}\right) x_{2}\left(\omega_{2}-v_{0} b_{2}\right)+\left(d_{2} / b_{2}\right)\left(\left(\omega_{2}-v_{0} b_{2}\right)^{2}+2\left(\omega_{2}-v_{0} b_{2}\right) v_{0} b_{2}+v_{0}^{2} b_{2}^{2}\right)-\pi / 2\right)} d \mathbf{x .}
\end{aligned}
$$

Hence,

$$
\begin{aligned}
& L_{A_{1}, A_{2}}^{\mathbb{U}}\left\{\mathbb{M}_{\boldsymbol{\omega}_{0}} f\right\}(\boldsymbol{\omega})=e^{\mathbf{i}\left(\omega_{1}-u_{0} b_{1}\right) u_{0} d_{1}+\mathbf{i} d_{1} u_{0}^{2} b_{1} / 2} L_{A_{1}, A_{2}}^{\mathbb{U}}\left(\omega_{1}\right. \\
& \left.\quad-u_{0} b_{1}, \omega_{2}-v_{0} b_{2}\right) e^{\mathbf{j}\left(\omega_{2}-v_{0} b_{2}\right) v_{0} d_{2}+\mathbf{j} d_{2} v_{0}^{2} b_{2} / 2} \\
& =e^{\mathbf{i} \omega_{1} u_{0} d_{1}} e^{-\mathbf{i}\left(\left(2 b_{1} d_{1} u_{0}^{2}-b_{1} d_{1} u_{0}^{2}\right) / 2\right)} L_{A_{1}, A_{2}}^{\mathbb{Q}}\left(\omega_{1}-u_{0} b_{1}, \omega_{2}\right. \\
& \left.-v_{0} b_{2}\right) e^{\mathbf{j} \omega_{2} v_{0} d_{2}} e^{-\mathbf{j}\left(\left(2 b_{2} d_{2} v_{0}^{2}-b_{2} d_{2} v_{0}^{2}\right) / 2\right)} \\
& =e^{\mathbf{i} \omega_{1} u_{0} d_{1}} e^{-\mathbf{i}\left(b_{1} d_{1} u_{0}^{2} / 2\right)} L_{A_{1}, A_{2}}^{\mathbb{1}}\left(\omega_{1}-u_{0} b_{1}, \omega_{2}-v_{0} b_{2}\right) \\
& \cdot e^{\mathbf{j} \omega_{2} v_{0} d_{2}} e^{-\mathbf{j}\left(b_{2} d_{2} v_{0}^{2} / 2\right)} .
\end{aligned}
$$

This is desired result.
Theorem 13 (time-frequency shift). If quaternion function $f \in L^{2}\left(\mathbb{R}^{2} ; \mathbb{H}\right)$, then one gets

$$
\begin{aligned}
& L_{A_{1}, A_{2}}^{\mathbb{W}}\left\{\mathbb{M}_{\boldsymbol{\omega}_{0}} \tau_{\mathbf{k}} f\right\}(\boldsymbol{\omega})=L_{A_{1}, A_{2}}^{\mathbb{W}}\left\{e^{\mathbf{i} x_{1} u_{0}} f(\mathbf{x}-\mathbf{k}) e^{\mathbf{j} x_{2} v_{0}}\right\} \\
& \cdot(\boldsymbol{\omega}) \\
& =e^{-\mathbf{i}\left(a_{1} c_{1} k_{1}^{2}+b_{1} d_{1} u_{0}^{2}\right) / 2+\mathbf{i}\left(c_{1} k_{1}+d_{1} u_{0}\right) \omega_{1}-\mathbf{i} b_{1} c_{1} k_{1} u_{0}} L_{A_{1}, A_{2}}^{\mathbb{U}}\{f\} \\
& \cdot\left(\omega_{1}-a_{1} k_{1}-u_{0} b_{1}, \omega_{2}-a_{2} k_{2}-v_{0} b_{2}\right) \\
& \cdot e^{-\mathbf{j}\left(a_{2} c_{2} k_{2}^{2}+b_{2} d_{2} v_{0}^{2}\right) / 2+\mathbf{j}\left(c_{2} k_{2}+d_{2} v_{0}\right) \omega_{2}-\mathbf{j} b_{2} c_{2} k_{2} v_{0}} .
\end{aligned}
$$

Proof. The proof directly follows from two previous theorems.

The above properties of the QLCT are summarized in Table 1. 
TABLE 1: Properties of the QLCT of $f, g \in L^{2}\left(\mathbb{R}^{2} ; \mathbb{H}\right)$, where $\alpha, \beta, \alpha^{\prime}, \beta^{\prime} \in \mathbb{U}$ are constants and $\boldsymbol{\omega}_{0}=u_{0} \mathbf{e}_{1}+v_{0} \mathbf{e}_{2} \in \mathbb{R}^{2}$.

\begin{tabular}{|c|c|c|}
\hline Property & Quaternion func. & QLCT \\
\hline Left linearity & $\alpha f+\beta g$ & $\alpha L_{A_{1}, A_{2}}^{\mathbb{\#}}\{f\}(\boldsymbol{\omega})+\beta L_{A_{1}, A_{2}}^{\mathbb{Z}}\{g\}(\boldsymbol{\omega})$ \\
\hline Right linearity & $f \alpha^{\prime}+g \beta^{\prime}$ & $L_{A_{1}, A_{2}}^{\mathbb{Q}}\{f\}(\boldsymbol{\omega}) \alpha^{\prime}+L_{A_{1}, A_{2}}^{\mathbb{W}}\{g\}(\boldsymbol{\omega}) \beta^{\prime}$ \\
\hline Shift & $f(\mathbf{x}-\mathbf{k})$ & $e^{-\mathbf{i} a_{1} c_{1} k_{1}^{2} / 2+\mathbf{i} c_{1} k_{1} \omega_{1}} L_{A_{1}, A_{2}}^{\mathbb{W}}\{f\}\left(\omega_{1}-a_{1} k_{1}, \omega_{2}-a_{2} k_{2}\right) e^{-\mathbf{j} a_{2} c_{2} k_{2}^{2} / 2+\mathbf{j} c_{2} k_{2} \omega_{2}}$ \\
\hline Modulation & $e^{\mathrm{i} x_{1} u_{0}} f(\mathbf{x}) e^{\mathbf{j} x_{2} v_{0}}$ & $e^{-\mathrm{i} b_{1} d_{1} u_{0}^{2} / 2+\mathrm{i} d_{1} u_{0} \omega_{1}} L_{A_{1}, A_{2}}^{\mathbb{N}}\{f\}\left(\omega_{1}-u_{0} b_{1}, \omega_{2}-v_{0} b_{2}\right) e^{-\mathrm{j} b_{2} d_{2} v_{0}^{2} / 2+\mathrm{j} d_{2} v_{0} \omega_{2}}$ \\
\hline Time-frequency & $e^{\mathbf{i} x_{1} u_{0}} f(\mathbf{x}-\mathbf{k}) e^{\mathbf{j} x_{2} v_{0}}$ & $\begin{array}{l}e^{-\mathbf{i}\left(a_{1} c_{1} k_{1}^{2}+b_{1} d_{1} u_{0}^{2}\right) / 2+\mathbf{i}\left(c_{1} k_{1}+d_{1} u_{0}\right) \omega_{1}-\mathbf{i} b_{1} c_{1} k_{1} u_{0}} L_{A_{1}, A_{2}}^{\boxplus}\{f\}\left(\omega_{1}-a_{1} k_{1}-u_{0} b_{1}, \omega_{2}-a_{2} k_{2}-v_{0} b_{2}\right) \\
\cdot e^{-\mathbf{j}\left(a_{2} c_{2} k_{2}^{2}+b_{2} d_{2} v_{0}^{2}\right) / 2+\mathbf{j}\left(c_{2} k_{2}+d_{2} v_{0}\right) \omega_{2}-\mathbf{j} b_{2} c_{2} k_{2} v_{0}}\end{array}$ \\
\hline Gaussian function & $e^{-\left(k_{1} x_{1}^{2}+k_{2} x_{2}^{2}\right)}$ & $\frac{1}{\sqrt{a_{1}+2 k_{1} b_{1}} \mathbf{i}} e^{\left(\omega_{1}^{2} / 2\right)\left(\left(c_{1}+2 k_{1} \mathbf{i} d_{1}\right) /\left(2 k_{1} b_{1}-\mathbf{i} a_{1}\right)\right)} \frac{1}{\sqrt{a_{2}+2 k_{2} b_{2} \mathbf{j}}} e^{\left(\omega_{2}^{2} / 2\right)\left(\left(c_{2}+2 k_{2} \mathbf{j} d_{2}\right) /\left(2 k_{2} b_{2}-\mathbf{j} a_{2}\right)\right)}$ \\
\hline
\end{tabular}

\section{Heisenberg Uncertainty Principle for QLCT}

The classical uncertainty principle of harmonic analysis states that a nontrivial function and its Fourier transform cannot be sharply localized simultaneously. In quantum mechanics, the uncertainty principle asserts that one cannot at the same time be certain of the position and of the velocity of an electron (or any particle) [20]. Let us now give an alternative proof of the Heisenberg type uncertainty principle for the QLCT, which is recently studied in [8] (the uncertainty principle of the QCT was proved using the exponential form of a $2 \mathrm{D}$ quaternion function and proposed proof of this paper uses the relationship between the QFT and QLCT). However, before proceeding with the statement of this main result, we need to introduce the component-wise uncertainty principle for the QFT as follows (see [12] for more details).

Theorem 14 (the QFT component-wise uncertainty principle). Suppose that $f \in L^{1}\left(\mathbb{R}^{2} ; \mathbb{M}\right) \cap L^{2}\left(\mathbb{R}^{2} ; \mathbb{H}\right)$. If $\partial f / \partial x_{k}$ and $\omega_{k}\left(\partial f / \partial x_{k}\right) \in L^{2}\left(\mathbb{R}^{2} ; \mathbb{H}\right)$, then one has

$$
\begin{gathered}
\int_{\mathbb{R}^{2}} x_{k}^{2}|f(\mathbf{x})|^{2} d \mathbf{x} \int_{\mathbb{R}^{2}} \omega_{k}^{2}\left|\mathscr{F}_{q}\{f\}(\boldsymbol{\omega})\right|^{2} d \boldsymbol{\omega} \\
\geq \frac{1}{4}\left(\int_{\mathbb{R}^{2}}|f(\mathbf{x})|^{2} d \mathbf{x}\right)^{2}, \quad k=1,2 .
\end{gathered}
$$

The generalization of the above uncertainty principle to the the QLCT domain is given by the following theorem (for more detailed information, see [8]).

Theorem 15 (the QLFT component-wise uncertainty principle). Assume that $f \in L^{1}\left(\mathbb{R}^{2} ; \mathbb{H}\right) \cap L^{2}\left(\mathbb{R}^{2} ; \mathbb{H}\right), \partial f / \partial x_{k} \in$ $L^{2}\left(\mathbb{R}^{2} ; \mathbb{H}\right)$ and that $L_{A_{1}, A_{2}}^{\mathbb{Q}}\{f\}, \omega_{k} L_{A_{1}, A_{2}}^{\mathbb{M}}\{f\} \in L^{2}\left(\mathbb{R}^{2} ; \mathbb{U}\right), k=$ 1,2. Then, the following inequality holds:

$$
\begin{gathered}
\int_{\mathbb{R}^{2}} x_{k}^{2}|f(\mathbf{x})|^{2} d \mathbf{x} \int_{\mathbb{R}^{2}} \omega_{l}^{2}\left|L_{A_{1}, A_{2}}^{\mathbb{\Perp}}\{f\}(\boldsymbol{\omega})\right|^{2} d \boldsymbol{\omega} \\
\geq \frac{b_{k}^{2}}{4}\left(\int_{\mathbb{R}^{2}}|f(\mathbf{x})|^{2} d \mathbf{x}\right)^{2}, \quad k=1,2 .
\end{gathered}
$$

Proof. Substituting the quaternion function $f$ by $g_{f}$ defined by (21) on both sides of (62), we easily obtain

$$
\begin{aligned}
& \int_{\mathbb{R}^{2}} x_{k}^{2}\left|g_{f}(\mathbf{x})\right|^{2} d \mathbf{x} \int_{\mathbb{R}^{2}} \omega_{k}^{2}\left|\mathscr{F}_{q}\left\{g_{f}\right\}(\boldsymbol{\omega})\right|^{2} d \boldsymbol{\omega} \\
& \quad \geq \frac{1}{4}\left(\int_{\mathbb{R}^{2}}\left|g_{f}(\mathbf{x})\right|^{2} d \mathbf{x}\right)^{2} .
\end{aligned}
$$

Now setting $\boldsymbol{\omega}=\boldsymbol{\omega} / \mathbf{b}$, we further have

$$
\begin{gathered}
\int_{\mathbb{R}^{2}} x_{k}^{2}\left|\frac{e^{-\mathbf{i}(\pi / 4)}}{\sqrt{b_{1}}} \tilde{f}(\mathbf{x}) \frac{e^{-\mathbf{j}(\pi / 4)}}{\sqrt{b_{2}}}\right|^{2} d \mathbf{x} \\
\cdot \int_{\mathbb{R}^{2}} \frac{\omega_{k}^{2}}{b_{k}^{2}}\left|\mathscr{F}_{q}\left\{g_{f}\right\}\left(\frac{\boldsymbol{\omega}}{\mathbf{b}}\right)\right|^{2} d \frac{\boldsymbol{\omega}}{\mathbf{b}} \\
\geq \frac{1}{4}\left(\int_{\mathbb{R}^{2}}\left|\frac{e^{-\mathbf{i}(\pi / 4)}}{\sqrt{b_{1}}} \tilde{f}(\mathbf{x}) \frac{e^{-\mathbf{i}(\pi / 4)}}{\sqrt{b_{2}}}\right|^{2} d \mathbf{x}\right)^{2},
\end{gathered}
$$

and thus

$$
\begin{gathered}
\int_{\mathbb{R}^{2}} \frac{x_{k}^{2}}{\left|b_{1} b_{2}\right|^{2}}|\tilde{f}(\mathbf{x})|^{2} d \mathbf{x} \int_{\mathbb{R}^{2}} \frac{\omega_{k}^{2}}{b_{k}^{2}}\left|\mathscr{F}_{q}\left\{g_{f}\right\}\left(\frac{\boldsymbol{\omega}}{\mathbf{b}}\right)\right|^{2} d \boldsymbol{\omega} \\
\geq \frac{1}{4\left|b_{1} b_{2}\right|^{2}}\left(\int_{\mathbb{R}^{2}}|\widetilde{f}(\mathbf{x})|^{2} d \mathbf{x}\right)^{2} .
\end{gathered}
$$

Hence,

$$
\begin{aligned}
& \int_{\mathbb{R}^{2}} \frac{x_{k}^{2}}{\left|b_{1} b_{2}\right|^{2}}\left|e^{\mathbf{i}\left(a_{1} / 2 b_{1}\right) x_{1}^{2}} f(\mathbf{x}) e^{\mathbf{j}\left(a_{2} / 2 b_{2}\right) x_{2}^{2}}\right|^{2} d \mathbf{x} \\
& \cdot \int_{\mathbb{R}^{2}} \frac{\omega_{k}^{2}}{b_{k}^{2}}\left|\mathscr{F}_{q}\left\{g_{f}\right\}\left(\frac{\boldsymbol{\omega}}{\mathbf{b}}\right)\right|^{2} d \boldsymbol{\omega} \\
& \geq \frac{1}{4\left|b_{1} b_{2}\right|^{2}}\left(\int_{\mathbb{R}^{2}}\left|e^{\mathbf{i}\left(a_{1} / 2 b_{1}\right) x_{1}^{2}} f(\mathbf{x}) e^{\mathbf{j}\left(a_{2} / 2 b_{2}\right) x_{2}^{2}}\right|^{2} d \mathbf{x}\right)^{2} .
\end{aligned}
$$


By inserting (23) into (66), we immediately obtain

$$
\begin{aligned}
& \int_{\mathbb{R}^{2}} \frac{x_{k}^{2}}{\left|b_{1} b_{2}\right|^{2}}\left|e^{\mathbf{i}\left(a_{1} / 2 b_{1}\right) x_{1}^{2}} f(\mathbf{x}) e^{\mathbf{j}\left(a_{2} / 2 b_{2}\right) x_{2}^{2}}\right|^{2} d \mathbf{x} \\
& \cdot \int_{\mathbb{R}^{2}} \frac{\omega_{k}^{2}}{b_{k}^{2}} \mid e^{-\mathbf{i}\left(d_{1} / 2 b_{1}\right) \omega_{1}^{2}} L_{A_{1}, A_{2}}^{\boxplus}\{f\}(\boldsymbol{\omega}) \\
& \left.\cdot e^{-\mathbf{j}\left(d_{2} / 2 b_{2}\right) \omega_{2}^{2}}\right|^{2} d \boldsymbol{\omega} \\
& \geq \frac{1}{4\left|b_{1} b_{2}\right|^{2}}\left(\int_{\mathbb{R}^{2}}\left|e^{\mathbf{i}\left(a_{1} / 2 b_{1}\right) x_{1}^{2}} f(\mathbf{x}) e^{\mathbf{j}\left(a_{2} / 2 b_{2}\right) x_{2}^{2}}\right|^{2} d \mathbf{x}\right)^{2} .
\end{aligned}
$$

Simplifying it gives

$$
\begin{gathered}
\int_{\mathbb{R}^{2}} x_{k}^{2}|f(\mathbf{x})|^{2} d \mathbf{x} \int_{\mathbb{R}^{2}} \omega_{k}^{2}\left|L_{A_{1}, A_{2}}^{\mathbb{\boxplus}}\{f\}(\boldsymbol{\omega})\right|^{2} d \boldsymbol{\omega} \\
\geq \frac{b_{k}^{2}}{4}\left(\int_{\mathbb{R}^{2}}|f(\mathbf{x})|^{2} d \mathbf{x}\right)^{2} .
\end{gathered}
$$

This finishes the proof of theorem.

It is not difficult to check that directional uncertainty principle for the QFT takes the following form (cf. [21, 22]).

Theorem 16. Suppose that $f \in L^{1}\left(\mathbb{R}^{2} ; \mathbb{H}\right) \cap L^{2}\left(\mathbb{R}^{2} ; \mathbb{H}\right)$. If $\partial f / \partial x_{k}$ and $\omega_{k}\left(\partial f / \partial x_{k}\right) \in L^{2}\left(\mathbb{R}^{2} ; \mathbb{H}\right)$, then one has

$$
\begin{gathered}
\int_{\mathbb{R}^{2}}|\mathbf{x}|^{2}|f(\mathbf{x})|^{2} d \mathbf{x} \int_{\mathbb{R}^{2}}|\boldsymbol{\omega}|^{2}\left|\mathscr{F}_{q}\{f\}(\boldsymbol{\omega})\right|^{2} d \boldsymbol{\omega} \\
\geq\left(\int_{\mathbb{R}^{2}}|f(\mathbf{x})|^{2} d \mathbf{x}\right)^{2} .
\end{gathered}
$$

Proceeding as in the proof of Theorem 15, we obtain the QLCT directional uncertainty principle as follows.

Theorem 17. Suppose that $f \in L^{1}\left(\mathbb{R}^{2} ; \mathbb{U}\right) \cap L^{2}\left(\mathbb{R}^{2} ; \mathbb{H}\right)$ and $L_{A_{1}, A_{2}}^{\mathbb{U}}\{f\}$ and $|\boldsymbol{\omega}|^{2} L_{A_{1}, A_{2}}^{\mathbb{U}}\{f\} \in L^{2}\left(\mathbb{R}^{2} ; \mathbb{H}\right)$. Then the following inequality is satisfied:

$$
\begin{aligned}
& \int_{\mathbb{R}^{2}}|\mathbf{x}|^{2}|f(\mathbf{x})|^{2} d \mathbf{x} \int_{\mathbb{R}^{2}}|\boldsymbol{\omega}|^{2}\left|L_{A_{1}, A_{2}}^{\boxplus 1}\{f\}(\boldsymbol{\omega})\right|^{2} d \boldsymbol{\omega} \\
& \quad \geq|\mathbf{b}|^{2}\left(\int_{\mathbb{R}^{2}}|f(\mathbf{x})|^{2} d \mathbf{x}\right)^{2} .
\end{aligned}
$$

\section{Conflict of Interests}

The authors declare that there is no conflict of interests regarding the publication of this paper.

\section{Acknowledgments}

The first author is partially supported by Hibah Penelitian Kompetisi Internal Tahun 2014 (no. 1454/UN4.20/PL.9/2014) from the Hasanuddin University, Indonesia. The second author is partially supported by JSPS. KAKENHI (C)25400202 of Japan.

\section{References}

[1] N. Goel and K. Singh, "A modified convolution and product theorem for the linear canonical transform derived by representation transformation in quantum mechanics," International Journal of Applied Mathematics and Computer Science, vol. 23, no. 3, pp. 685-695, 2013.

[2] P. Dang, G.-T. Deng, and T. Qian, "A tighter uncertainty principle for linear canonical transform in terms of phase derivative," IEEE Transactions on Signal Processing, vol. 61, no. 21, pp. 5153-5164, 2013.

[3] H. M. Ozaktas, Z. Zalevsky, and M. A. Kutay, The Fractional Fourier Transform with Application in Optics and Signal Processing, Willey, New York, NY, USA, 2001.

[4] J. Zhao, R. Tao, Y.-L. Li, and Y. Wang, "Uncertainty principles for linear canonical transform," IEEE Transactions on Signal Processing, vol. 57, no. 7, pp. 2856-2858, 2009.

[5] X. Guanlai, W. Xiaotong, and Y. Xiaogang, "On uncertainty principle for the linear canonical transform of complex signals," IEEE Transactions on Signal Processing, vol. 58, no. 9, pp. 49164918, 2010.

[6] J. Shi, X. Liu, and N. Zhang, "On uncertainty principles for linear canonical transform of complex signals via operator methods," Signal, Image and Video Processing, vol. 8, no. 1, pp. 85-93, 2014.

[7] B. Hu, Y. Zhou, L. D. Lie, and J. Y. Zhang, "Polar linear canonical transform in quaternion domain," Journal of Information Hiding and Multimedia Signal Processing, vol. 6, no. 6, pp. 1185-1193, 2015.

[8] K. I. Kou, J.-Y. Ou, and J. Morais, "On uncertainty principle for quaternionic linear canonical transform," Abstract and Applied Analysis, vol. 2013, Article ID 725952, 14 pages, 2013.

[9] K. I. Kou and J. Morais, "Asymptotic behaviour of the quaternion linear canonical transform and the Bochner-Minlos theorem," Applied Mathematics and Computation, vol. 247, no. 15, pp. 675-688, 2014.

[10] Y. Yang and K. I. Kou, "Uncertainty principles for hypercomplex signals in the linear canonical transform domains," Signal Processing, vol. 95, pp. 67-75, 2014.

[11] M. Bahri, "Quaternion linear canonical transform application," Global Journal of Pure and Applied Mathematics, vol. 11, no. 1, pp. 19-24, 2015.

[12] M. Bahri, E. S. Hitzer, A. Hayashi, and R. Ashino, "An uncertainty principle for quaternion Fourier transform," Computers \& Mathematics with Applications, vol. 56, no. 9, pp. 2398-2410, 2008.

[13] E. M. Hitzer, "Directional uncertainty principle for quaternion Fourier transform," Advances in Applied Clifford Algebras, vol. 20, no. 2, pp. 271-284, 2010.

[14] T. Bülow, Hypercomplex spectral signal representations for the processing and analysis of images [Ph.D. thesis], University of Kiel, Kiel, Germany, 1999.

[15] M. Bahri, R. Ashino, and R. Vaillancourt, "Convolution theorems for quaternion Fourier transform: properties and applications," Abstract and Applied Analysis, vol. 2013, Article ID 162769, 10 pages, 2013.

[16] E. M. Hitzer, "Quaternion Fourier transform on quaternion fields and generalizations," Advances in Applied Clifford Algebras, vol. 17, no. 3, pp. 497-517, 2007.

[17] Q. Xiang and K.-Y. Qin, "On the relationship between the linear canonical transform and the Fourier transform," in Proceedings 
of the 4th International Congress on Image and Signal Processing (CISP '11), pp. 2214-2217, IEEE, Shanghai, China, October 2011.

[18] A. I. Zayed, "On the relationship between the Fourier and fractional Fourier transforms," IEEE Signal Processing Letters, vol. 3, no. 12, pp. 310-311, 1996.

[19] M. Bahri, Zulfajar, and R. Ashino, "Convolution and correlation theorem for linear canonical transform and properties," Information B, vol. 17, no. 6, pp. 2509-2521, 2014.

[20] W. Weyl, The Theory of Groups and Quantum Mechanics, Dover, New York, NY, USA, 2nd edition, 1950.

[21] L.-P. Chen, K. I. Kou, and M.-S. Liu, "Pitt's inequality and the uncertainty principle associated with the quaternion Fourier transform," Journal of Mathematical Analysis and Applications, vol. 423, no. 1, pp. 681-700, 2015.

[22] Y. Yang, P. Dang, and T. Qian, “Tighter uncertainty principles based on quaternion Fourier transform," Advances in Applied Clifford Algebras, 2015. 


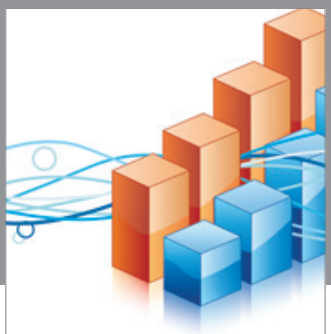

Advances in

Operations Research

vatem alat4

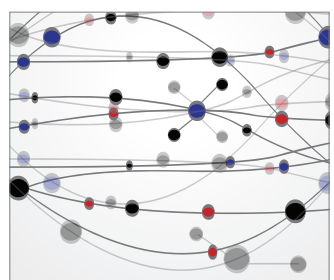

\section{The Scientific} World Journal
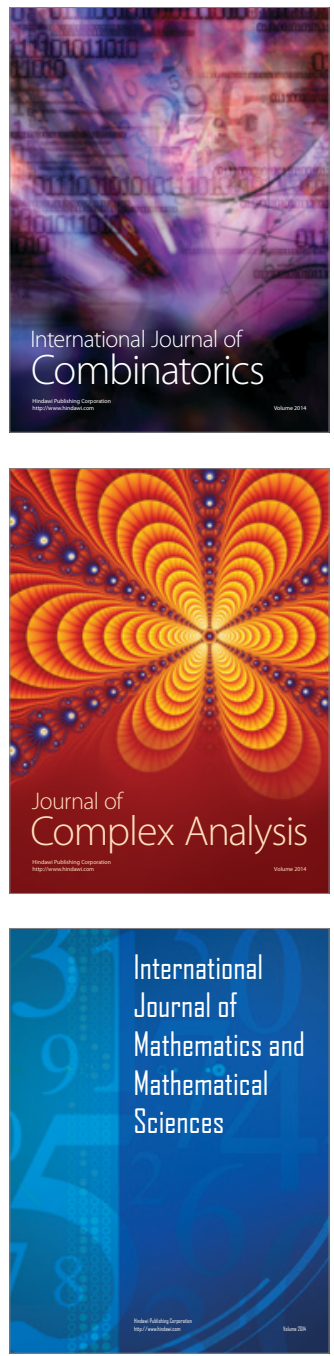
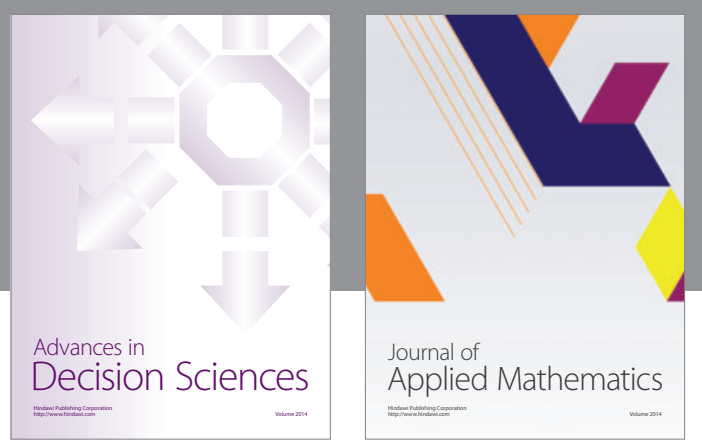

Algebra

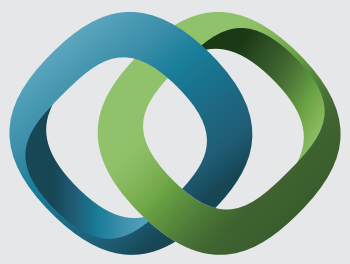

\section{Hindawi}

Submit your manuscripts at

http://www.hindawi.com
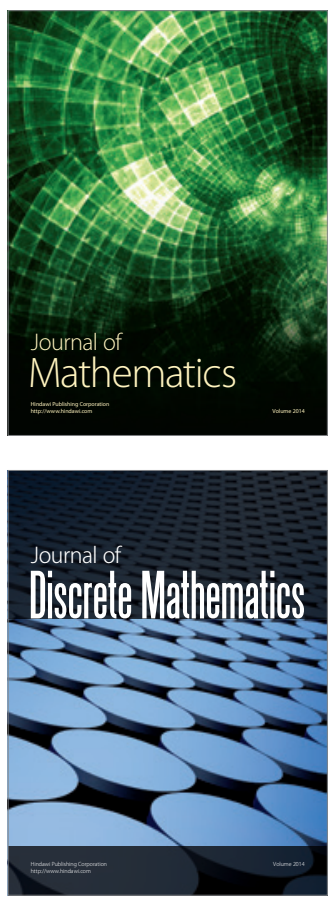

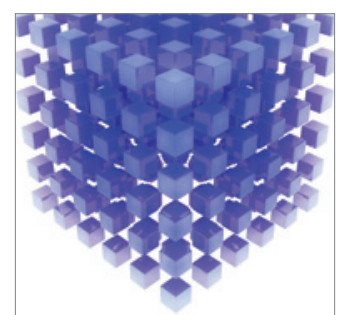

Mathematical Problems in Engineering
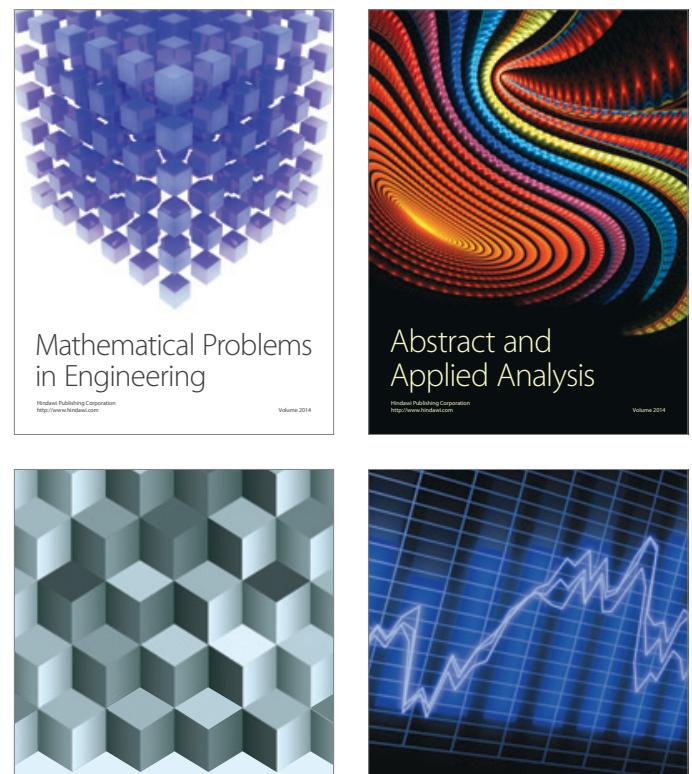

Journal of

Function Spaces

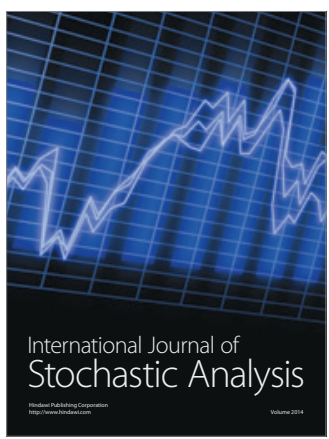

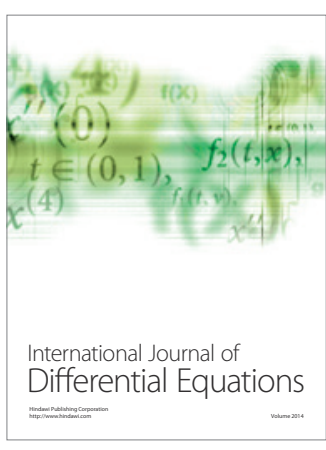
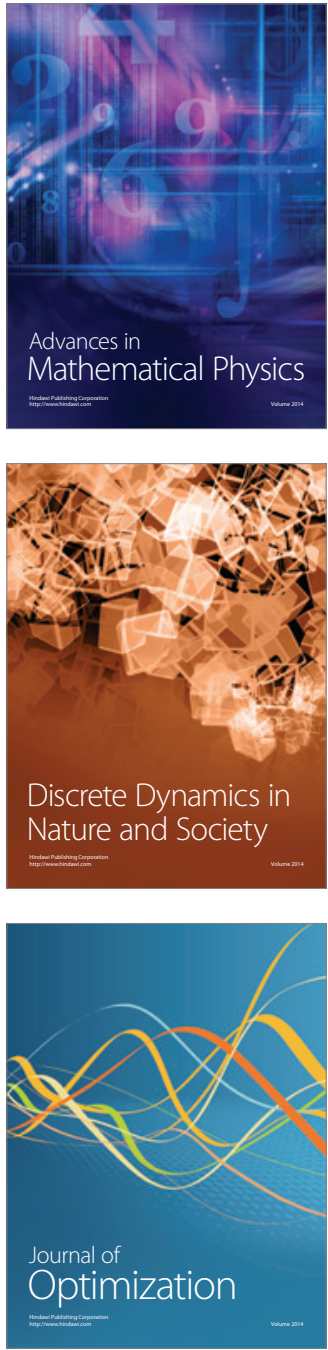